\title{
LA DECISIÓN DEL ELECTOR: PARTIDISMO, RACIONALIDAD-ECONOMÍA Y ESTRUCTURA SOCIAL
}

\author{
José Luis Sáez Lozano
}

Universidad de Granada

\begin{abstract}
RESUMEN
En este estudio planteamos la necesidad de analizar la decisión de los electores desde una perspectiva comprehensiva. Hasta ahora, la teoría del comportamiento electoral ofrecía explicaciones parciales sobre cómo los electores orientan su decisión final: la identificación partidista, la visión racionalista y los planteamientos de la estructura social.

Nuestro objetivo es especificar una función de voto que integre estos tres enfoques. Para ello hemos de asumir que los votantes pueden elegir entre un conjunto de opciones políticas, y que la decisión observada revela cuál es la alternativa que le proporciona mayor utilidad. Al haber más de dos partidos, nos vemos obligados a plantear un modelo de elección discreta múltiple. De acuerdo con las características de la información disponible, hemos especificado un modelo logit multinomial.

A partir de las funciones de voto estimadas, elaboramos un decálogo del voto en la democracia española, con el fin de sintetizar los principales rasgos que han caracterizado el comportamiento electoral de los españoles a lo largo de este periodo.
\end{abstract}

El análisis de la decisión individual de los electores es una nueva visión del comportamiento electoral que consiste en investigar las razones últimas que llevan a los votantes a optar por una determinada opción política. Una de las ventajas que ofrece este enfoque es que permite incluir variables explicativas de distinta índole: ideológicas, partidistas, situacionales, psicopolíticas... 
En ese sentido, nuestro objetivo en este trabajo es reafirmar la visión comprehensiva del comportamiento electoral, apoyándonos en el análisis empírico de la función de voto en la democracia española. De este estudio se infiere que es necesario analizar la decisión final del elector desde la triple perspectiva que ofrece la identificación partidista, la teoría racionalista y el planteamiento de la estructura social; y aportamos como prueba el caso español, que evidencia cómo este modo de abordar el estudio de la orientación del voto es más riguroso que los enfoques parciales y segmentados.

Con el fin de alcanzar este objetivo hemos organizado este artículo en cuatro apartados, más uno dedicado a exponer las principales conclusiones que se extraen de este estudio. En el epígrafe siguiente argumentamos por qué es necesario analizar el comportamiento electoral desde una perspectiva comprehensiva. Este apartado quedaría inconcluso si no revisamos a continuación, de forma sucinta, los diferentes planteamientos del comportamiento electoral (apartado 2). Tras examinar los aspectos conceptuales y teóricos de la decisión electoral, presentamos los fundamentos de la función de voto multinomial, que vamos a utilizar para identificar las razones que han determinado la decisión electoral de los españoles (epígrafe 3). En el penúltimo apartado de este estudio presentamos los diez rasgos que han distinguido y caracterizado el comportamiento electoral de los españoles en los comicios generales de 1982, 1986, 1993 y $1996^{1}$.

\section{DESIDERATUM: LA NECESIDAD DE UN ENFOQUE COMPREHENSIVO}

Tal y como hemos reseñado en la introducción, el análisis del comportamiento electoral se ha planteado desde muy diferentes perspectivas teóricas. Esta pluralidad de enfoques no es inmune al hecho de que estamos ante un programa de investigación complejo, que exige una visión comprehensiva a la hora de analizar la decisión final de los electores. En ese sentido, este enfoque novedoso debiera de convertirse en un desideratum, máxime cuando se trata de una investigación empírica².

Existe un amplio consenso, a nivel conceptual, de que estos tres planteamientos son complementarios y, además, pueden integrarse en una función de voto multivariante que combina las principales variables que explican la decisión final del elector. Uno de los primeros trabajos que se publicaron en esta línea de pensamiento fue el de Kramer (1971), quien planteó una ecuación

${ }^{1}$ No hemos incluido las convocatorias a las urnas de 1977 y 1979, ya que en el Banco de Datos del Centro de Investigaciones Sociológicas (CIS), que es la única fuente estadística (pública y privada) que facilita esta información, no hay encuestas postelectorales de estos comicios.

${ }^{2}$ En nuestro caso, nos planteamos analizar la función de voto de los españoles en la democracia. 
multinomial de elección discreta para explicar el voto, como una función de diferentes atributos psicológicos y sociológicos del votante. Este ámbito de investigación experimentó un extraordinario progreso con las aportaciones posteriores de Lewis-Beck (1990), Álvarez y Nagle (1995), Whitten y Palmer (1996)...

Desde la perspectiva metodológica también se ha abordado el análisis del comportamiento electoral de diferentes formas. Los defensores de la identificación partidista suponen que los votantes realizan un análisis inductivo para identificarse con un candidato y/o partido, adoptan unas actitudes hacia éstos e incluso llegan a internalizar una serie de valores que definen la orientación de su decisión. Los estudiosos de la teoría racionalista han apostado por el deductivismo: el votante opta por aquella opción que satisface sus objetivos, ya que ello le permite maximizar su bienestar individual.

A la hora de contrastar los postulados de estas tres visiones del comportamiento, también se han utilizado métodos diferentes. Los estudios de la identificación partidista suelen plantear un esquema de ecuaciones estructurales a la hora de modelizar la decisión final de los electores ${ }^{3}$; por el contrario, los análisis que se enmarcan dentro de la teoría racionalista se aproximan a este fenómeno mediante modelos uniecuacionales; si bien es cierto que en el caso de la contrastación de la hipótesis downsiana se han utilizado dos vías diferentes: las macrorregresiones, cuando se intenta explicar la decisión de colectivos de votantes (por distritos...), y la microrregresión, si se utilizan datos a nivel de individuos $^{4}$. Para analizar la función de voto de la democracia española planteamos un modelo que está en sintonía con las propuestas de Riker y Ordeshook (1968), Ashenfelter y Kelley (1975), Lewis-Beck (1990), Matsusaka y Palda (1993)...

3 Vid. B. I. Page y R. A. Brody (1972), Policy voting and the electoral process: The Vietnam war issues, pp. 979-995; A. S. Goldberg (1966), Dicerning a causal pattern among data on voting behavior, pp. 913-922; J. E. Jackson (1975), Issues, party choice and presidential votes, pp. 161186; M. A. Schuman y G. M. Pomper (1975), Variability in electoral behavior: Longitudinal perspectives from causal modeling, pp. 1-18; E. Declercq, T. L. Hurley y N. R. Luttberg (1975), Voting in American Presidential elections: 1956-1972, pp. 913-922; Ch. H. Achen (1975), Mass political attitudes and the survey response, pp. 1218-1231; G. B. Markus y P. E. Converse (1979), A dinamic simultaneous equation model of electoral choice, pp. 1055-1070; y B. I. Page y C. C. Jones (1979), Reciprocal effects of policy preferences, party loyalties and the vote, pp. 1071-1089.

${ }^{4}$ Los estudios empíricos que contrastan la hipótesis de Downs han suscitado, por extensión, un debate que se conoce como la falacia ecológica del voto; es decir, se discute si, a partir de los análisis con datos del comportamiento electoral agregados (por distritos...), pueden inferirse conclusiones respecto a la decisión individual del votante de ir a votar o no. Kramer (1983) profundizó en esta cuestión y concluyó que no era posible obviar esta cuestión de la agregación dentro del comportamiento electoral, ya que ello desvirtuaba las conclusiones a nivel individual; por otro lado, J. G. Matsuaka y F. Palda (1993) evidenciaron que, en el caso canadiense (elecciones nacionales de 1974, 1979 y 1980), lo correcto era contrastar la hipótesis a través de una microrregresión. Simultáneamente, han ido apareciendo otros estudios que plantearon una macrorregresión para contrastar la hipótesis de Downs, obviando los efectos perniciosos que introducen los datos agregados: Barzel y Silbergerg (1973), Durden y Gaynor (1987)... 
En el análisis del comportamiento electoral también se han utilizado distintas técnicas cuantitativas: Nie, Verba y Petrocik (1976) empleaban el análisis de varianza para explicar los cambios en el voto presidencial en los EE.UU. entre 1956 y 1960; Shapiro (1969) utilizó el análisis factorial en el estudio que realizó para explicar la intención de voto de los cinco políticos que en 1968 hicieron campaña para ser nominados candidatos a la Presidencia de EE.UU.5; por último, Lewis-Beck (1990), Matsusaka y Palda (1993) y otros aplicaron modelos logits para aproximarse al estudio del voto económico en Gran Bretaña, Francia, Alemania e Italia, o descubrir cómo los canadienses adoptaron su decisión en las elecciones nacionales de 1974, 1979 y 1980.

\section{EL COMPORTAMIENTO ELECTORAL: PARTIDISMO, ECONOMÍA Y ESTRUCTURA SOCIAL}

Tal y como hemos adelantado en la introducción, los distintos modelos explicativos del comportamiento electoral se fundamentan en concepciones teóricas diferenciadas, e incluso a veces contrapuestas; sin embargo, la principal enseñanza que se extrae de los numerosos estudios empíricos realizados es que éstas no son tan incompatibles ${ }^{6}$. A partir de ahí, surge la necesidad de utilizar modelos comprehensivos que expliquen cómo adoptan su decisión final los electores. La función de voto que proponemos está en sintonía con esta nueva visión del comportamiento electoral, ya que sus variables explicativas reflejan los postulados de la identificación partidista, el racionalismo y la base social del voto ${ }^{7}$.

El argumento central de la teoría de la identificación partidista es que los votantes van adquiriendo, a lo largo de su proceso de socialización, afinidades con determinados partidos políticos. El nivel de simpatía del elector con las distintas fuerzas políticas o ideologías es el elemento que orientará el sentido del voto; se crea así un vínculo a largo plazo. Este enfoque contribuye a explicar el anclaje electoral, es decir, los factores que determinan la fidelidad de los votantes con un determinado partido o ideología política. En ese sentido, los realignment en el espectro político tan sólo se pueden producir cuando cambian las afinidades ideológicas o las actitudes políticas de los electores; y ello sólo puede suceder en el largo plazo, ya que es el periodo de tiempo en el que se producen los cambios generacionales ${ }^{8}$.

Frente a los planteamientos de la identificación partidista emergieron los estudios que intentaban explicar el voto como una decisión racional. El trabajo pionero de Downs recibió fuertes críticas, y ello explica por qué aparecieron las

Humphrey, Kennedy, McCarthy, Nixon y Rockefeller.

6 Vid. M. Harrop y W. L. Miller (1988), Elections and voters. A Comparative Introduction.

Vid. M. S. Lewis-Beck (1990), Economic and elections...

${ }^{8}$ Vid. N. H. Nie, J. R. Verba y J. R. Petrocik (1976), The changing American voter. 
propuestas del voto retro y prospectivo ${ }^{9}$, como respuesta a estas contestaciones. Posteriormente, surgieron los planteamientos racionalistas de la teoría espacial y direccional del voto. Enelow e Hinich (1984), a partir de la teoría económica del voto de Downs y del teorema del votante mediano de Black, plantearon que tanto los votantes como los partidos y/o candidatos se sitúan en un continuum bidimensional, que refleja las posiciones de ambos colectivos respecto a cada uno de los issues políticos; al final, el elector opta por la opción más próxima, es decir, aquella que le permite minimizar la distancia entre él y las distintas fuerzas políticas. Por el contrario, la teoría direccional del voto es una versión diferenciada de la espacial; en este caso, los votantes no eligen finalmente a aquellos partidos y/o candidatos más próximos, sino que optan por quienes defienden con mayor intensidad sus preferencias ${ }^{10}$.

No podemos olvidar la relevancia que ha adquirido la teoría del voto económico como un factor más del comportamiento electoral. Los gobiernos ganan o pierden elecciones en función de los resultados económicos derivados de su actuación política. Los votantes, que se suponen personas responsables, atribuyen los resultados de la política económica articulada (positivos y negativos) al(los) partido(s) que respalda(n) parlamentariamente al gobierno; de ahí que premien a aquellos políticos que en su acción de gobierno favorecen el auge económico y castiguen a quienes inducen crisis en el nivel de actividad ${ }^{11}$. Otra cuestión es identificar los argumentos que utiliza el elector para dictaminar su sentencia de premio-castigo ${ }^{12}$.

Además de estos planteamientos, dentro de la teoría racional del voto hay diversos estudios que analizan la influencia de issues como las cualidades, competencia e imagen del candidato en la decisión final del elector ${ }^{13}$.

La teoría del voto determinado por la estructura social enfatiza la importancia de los factores socioestructurales como variables explicativas del comportamiento electoral. Los grupos sociales pueden diferenciarse en base a variables tales como el género, la pertenencia a un determinado colectivo social, étnico, edad, religión...; y éstos, a su vez, permiten identificar a los votantes con deter-

9 Vid. W. H. Riker y P. Ordeshook (1968), A theory of the calculus of voting.

${ }^{10}$ Vid. G. Rabinowitz y S. E. MacDonald (1989), A directional theory of issue voting, pp. 93121.

${ }_{11}$ A este principio se le denomina hipótesis de la responsabilidad.

12 Vid. M. S. Lewis-Beck (1990), Economics and elections..., pp. 33-52. El autor plantea esta cuestión como si fuese uno de los grandes interrogantes que aún quedan por responder en el seno de la teoría económica del voto.

${ }_{13}$ Vid. M. J. Shapiro (1969), Rational political man: A synthesis of economic and social-psychological perspectives, pp. 1106-1119; M. F. Fiorina (1977), An outline for a Model of Party Choice, pp. 601-625; G. B. Markus y P. E. Converse (1979), A dynamic simultaneous equations model of electoral choice, pp. 1055-1070; B. I. Page y C. C. Jones (1979), Reciprocal effects of policy preferences, party loyalties and the vote, pp. 1071-1089; P. M. Sniderman, J. M. Glaser y R. Griffin (1990), Information and electoral choice, pp. 117-135; M. R. Rahn, J. H. Aldrich, E. Borgida y J. L. Sullivan (1990), A social-cognitive model of candidate appraisal, pp. 136-159; y S. Iyengar (1990), Shortcus to political knowledge: The role of selective, pp. 160-185. 
minados partidos. Ello explica por qué las fuerzas políticas son un medio de representación de los intereses del grupo, y las bases sociales de los partidos las conforman los electores. El proceso de formación de los grupos se explica históricamente por los cleavages políticos presentes en cada sociedad, que provocan la unión de los votantes (de cada fragmentación) en su acción política, ya que todos ellos están muy próximos y tienen un elevado grado de interrelación.

\section{LA FUNCIÓN DE VOTO EN DEMOCRACIA}

En este apartado exponemos los fundamentos teóricos de la función de voto que vamos a utilizar para explicar el comportamiento electoral de los españoles en la democracia. Asumimos que un votante $i$ puede elegir entre un conjunto de opciones políticas $j^{14}$; y que la decisión observada revela cuál es la alternativa que le proporciona mayor utilidad $U^{15}$. Así pues:

$$
U_{i j}=u_{i}\left(x, \beta_{j}\right) \quad \forall i=1,2, \ldots, n ; \quad \mathrm{j}=1,2, \ldots, l
$$

El vector $x$ es el conjunto de factores que determinan por qué el elector $i$ termina votando al partido $j\left(V_{i j}\right)^{16}$ :

Variables socioestructurales. A este grupo pertenecen:

$-e=$ La edad del elector.

$-s=$ El género.

- $c i=$ El estado civil.

- $n e=$ El nivel de educación del elector.

- $s p=$ El ámbito donde trabaja el elector (sector público o no).

- $a s=\mathrm{Si}$ el elector es asalariado o no.

- $d e=$ Si el elector está desempleado o no.

- $n a=$ Si el elector habita en las Comunidades Autónomas del País Vasco o Cataluña.

- $p e=$ Si el elector es pensionista o no.

$-h=$ El tamaño del hábitat en el que reside el elector.

${ }_{14}$ En nuestro caso, el votante puede elegir entre cinco opciones electorales (PNV, IU, PSOE, CiU y PP); excepto en las elecciones generales de 1982, donde, además de los partidos mencionados, incluimos a la UCD y el CDS; sin embargo, en los comicios de 1986, tan sólo aparece como opción adicional el CDS.

15 Ésta es una variable no observable.

${ }^{16}$ Hemos de reseñar que las variables explicativas que se mencionan a continuación no se incluyeron en todas las funciones de voto estimadas, ya que algunas de las encuestas postelectorales no incluían ítems que nos permitiesen medir la influencia de algunos factores explicativos del voto. 
- $f a=$ El número de hijos que componen la familia del elector.

$-y=$ El nivel de renta familiar del elector.

- $c s=$ La clase social subjetiva del elector, es decir, en la que se ubica el votante.

- $r e=$ La religión del elector.

- pre = El nivel de práctica religiosa del elector.

Variables situacionales y temas coyunturales. A este grupo pertenecen:

- $t v=$ La valoración que hace el elector de los debates televisados ${ }^{17}$.

- en $=\mathrm{La}$ valoración que merecen las encuestas preelectorales para el votante.

- $c=$ La percepción que tiene el elector de la campaña realizada por la fuerza política a la que votó.

- $l=$ La valoración que otorga el elector al líder político del partido elegido.

Factores ideológicos y variables psicopolíticas. A este grupo pertenecen:

- $i=$ La identificación partidista del elector.

- $d i=$ La distancia ideológica entre el elector y la opción electoral por la que optó el votante ${ }^{18}$.

Variables de carácter racionalista. A este grupo pertenecen:

- $p p p=$ La percepción (prospectiva) que posee el elector de la situación política del país.

- $p p e=$ La percepción (prospectiva) que posee el elector de la situación económica del país.

- $c g$ = Capacidad de gobierno del partido elegido.

Otras variables y factores. A este grupo pertenecen:

17 tv es una variable discreta, que refleja el seguimiento que hicieron los electores de los debates televisados entre José María Aznar y Felipe González en las elecciones generales de 1993.

${ }^{18}$ di es una variable continua, que representa la distancia ideológica (en términos nominales) entre el votante y el partido al que ha votado:

$$
d i_{i}^{j}=I_{i}-I^{j} \quad \forall i=1,2, \ldots, n ; \quad \mathrm{j}=1,2, \ldots, k
$$

siendo:

$-I_{i}=$ La posición ideológica del votante $i$ en el plano ideológico $I$.

- $I^{j}=$ La posición ideológica del partido $j$ en el plano ideológico $I$. 
- $r v=$ El recuerdo de voto, es decir, por qué opción electoral optó el votante en los comicios generales anteriores ${ }^{19}$.

- $v p=\mathrm{El}$ voto prospectivo, es decir, por qué opción electoral optaría el votante en los próximos comicios generales.

El vector $\beta_{j}$ refleja el impacto de $x$ sobre la probabilidad de que un votante $i$ opte finalmente por el partido $j\left(P_{i j}\right)$ :

$$
P_{i_{j}}=P\left(V_{i_{j}} \mid x\right)=P\left(U_{i j}>U_{i k}\right) \quad \forall i=1,2, \ldots, n ; \quad j=1,2, \ldots, k, \ldots, l
$$

siendo

$\beta_{e}, \beta_{r v}, \beta_{v p}, \beta_{v e}, \beta, \beta_{c}, \beta_{n e}, \beta_{s p}, \beta_{a s}, \beta_{c g}, \beta_{d e}, \beta_{n a}, \beta_{p e}, \beta_{b}, \beta_{f a}, \beta_{y}, \beta_{c s}, \beta_{r e}, \beta_{p r e}$ $\beta_{t v}, \beta_{e n}, \beta_{c}, \beta_{l}, \beta_{i}, \beta_{d i}, \beta_{p p e}, y \beta_{p p p}$, parámetros a estimar.

Al haber más de dos opciones electores $(j>2)$ nos vemos obligados a plantear un modelo de elección discreta múltiple: probabilidad lineal, logit o probit multinomial. De acuerdo con las características de la información disponible, lo más oportuno es plantear un modelo logit:

$$
P_{i_{j}}=\frac{e^{u_{j}}}{1+\sum_{j=1}^{l} e^{u_{j}}} \forall j=1,2, \ldots, l
$$

A partir de la expresión anterior, podemos deducir el modelo multinomial que determina de forma única la probabilidad de voto de cada opción electoral, y que garantiza que la suma de todas ellas es igual a la unidad:

$$
\left\{\begin{array}{c}
P_{i_{j}}=\frac{e^{u_{j}}}{1+\sum_{j=2}^{l} e^{u_{j}}} \\
P_{i_{1}}=\frac{1}{1+\sum_{j=2}^{l} e^{u_{j}}}
\end{array}\right.
$$

19 Se incluyó tanto el recuerdo de voto de los comicios generales $(r v g)$ como el de las autonómicas (rva). 
Esta función de voto se fundamenta sobre dos supuestos: 1) las distintas opciones electorales $j$ son independientes ${ }^{20}$, y 2) las diferentes alternativas $j$ no son fácilmente sustituibles entre sí. Además de estos supuestos, realizamos dos contrastes de hipótesis adicionales: 1) los errores del modelo de regresión subyacente son homocedásticos por grupos (opciones políticas) ${ }^{21}$, y 2) no se omiten variables explicativas significativas ${ }^{22}$.

Una vez estimado el modelo [4], podremos simular las probabilidades de voto para cada opción electoral en las distintas convocatorias analizadas y calcular sus elasticidades $(\varepsilon)$ ante un cambio en cualquiera de los factores determinantes del $\operatorname{voto}^{23}$. El valor de las elasticidades refleja cómo influyen los distintos factores explicativos del comportamiento electoral en la decisión final de los votantes.

\section{EL VOTO DE LOS ESPAÑOLES EN LA DEMOCRACIA: UN DECÁLOGO}

Desde el 15 de diciembre de 1976, fecha en que los españoles votaron mayoritariamente la Reforma Política, hasta nuestros días se han celebrado ocho elecciones a Cortes Generales y al Senado: 15 de junio de 1977, 1 de marzo de 1979, 28 de octubre de 1982, 22 de junio de 1986, 29 de octubre de 1989, 6 de junio de 1993 y 3 de marzo de $1996^{24}$. La UCD gobernó en minoría hasta 1982, ya que tras los comicios generales celebrados en octubre de ese año comenzó el ciclo gubernativo del PSOE. Los comicios generales de 1993 fueron muy competitivos, y ya se atisbaba en el horizonte electoral la alternancia popular. En las elecciones de 1996, el PP consiguió una mayoría relativa que le obligó a establecer acuerdos de legislatura con las tres fuerzas políticas de carácter nacionalista más respaldadas parlamentariamente: CiU, PNV y Coalición Canaria (CC).

Tal y como adelantamos en la introducción, los datos utilizados en este trabajo proceden de los sondeos postelectorales realizados por el CIS. En ese sentido, hemos de reseñar que no hemos podido medir la influencia de facto-

${ }^{20}$ Vid. W. H. Greene (1998), Análisis econométrico, pp. 791-792. Para aceptar o rechazar este supuesto utilizamos el contraste de Hausmann y McFadden (HM).

${ }^{21}$ Vid. W. H. Greene (1998), op. cit., pp. 758-766. Para aceptar o rechazar la ausencia de homocedasticidad por grupos utilizamos el contraste de multiplicadores de Lagrange (LM).

22 Vid. W. H. Greene (1998), op. cit., pp. 763-766. Consideramos dos alternativas: 1) que todos los parámetros del modelo fuesen igual a 0 , y 2) que el conjunto de coeficientes correspondientes a las variables que representan la identificación partidista, la teoría racional y los factores fuesen iguales a 0 . Para realizar estos contrastes utilizamos el test de Wald (W) y la razón de verosimilitud (LR).

${ }^{23}$ Para cada una de las variables explicativas hemos calculado las elasticidades medias en los distintos valores de la escala. Consideramos que dichas variables, excepto aquella que utilizamos para el cálculo de $\varepsilon$, toman el valor medio.

${ }^{24}$ El 12 de marzo de 2000 se celebraron los octavos comicios generales. 
res como la percepción retro y prospectiva de la situación política y económica del país, el voto estratégico y prospectivo, y otras variables de índole racional y de identificación partidista, ya que ninguna de las encuestas utilizadas incluía ítems que nos permitiesen cuantificar estas variables explicativas.

Antes de pasar a analizar la función de voto de los españoles en la democracia, considero que es oportuno señalar los aspectos más relevantes de la evaluación de los modelos estimados. Tal y como era previsible, no se puede aceptar la hipótesis de no nulidad para todos los parámetros estimados, con un nivel de significación igual o inferior al 0,05 (véanse los cuadros 1, 2, 3, 4 y 5). Por otra parte, en ninguna de las funciones de voto estimadas se aprecia la presencia de un error de especificación I: omisión colectiva de todas las variables explicativas, es decir, todos los coeficientes estimados de las variables de identificación partidista, racionalista y socioestructurales son iguales a 0 . En la línea de esto último, tampoco podemos concluir que puedan omitirse en bloque las variables explicativas correspondientes a cada uno de los grupos de factores explicativos del comportamiento electoral: els, situacionales, ideológicos y racionales.

Respecto a la hipótesis de independencia de las alternativas políticas (PCE/IU, PSOE, CDS, CiU, PNV y PP) irrelevantes, los test de HM certifican que podemos aceptar este supuesto. Además, las funciones de voto estimadas no presentan problemas de heterocedasticidad por colectivos (opciones o alternativas políticas), según se infiere del estadístico $\mathrm{ML}^{25}$.

${ }^{25}$ Véanse los cuadros $1,2,3,4$ y 5. 


\section{CUADRO 1}

Estimación de la función de voto en las elecciones generales de 1982

\begin{tabular}{|c|c|c|c|c|c|}
\hline Opción electoral & Variable & Coeficiente & $\begin{array}{l}\text { Desviación } \\
\text { estándar } \\
\text { coeficiente }\end{array}$ & T-Student & $\begin{array}{c}\text { Nivel } \\
\text { significación } \\
{\left[\text { Prob }|t|>x\left({ }^{*}\right)\right]}\end{array}$ \\
\hline \multirow{13}{*}{ PCE } & en & $-8,03 \mathrm{E}-02$ & 0,35793 & $-0,224$ & 0,82251 \\
\hline & $r v g$ & $2,84 \mathrm{E}+00$ & 0,3854 & 7,363 & 0,00001 \\
\hline & $h$ & $7,17 \mathrm{E}-07$ & $3,09 \mathrm{E}-07$ & 2,319 & 0,02041 \\
\hline & $d e$ & $-1,22 \mathrm{E}-02$ & $5,40 \mathrm{E}-01$ & $-0,023$ & 0,98201 \\
\hline & pe & $-5,49 \mathrm{E}-01$ & $8,64 \mathrm{E}-01$ & $-0,636$ & 0,52508 \\
\hline & $s$ & $3,39 \mathrm{E}-01$ & $3,47 \mathrm{E}-01$ & 0,976 & 0,32921 \\
\hline & na & $-4,30 \mathrm{E}-01$ & $3,80 \mathrm{E}-01$ & $-1,133$ & 0,25727 \\
\hline & as & $-1,05 \mathrm{E}+00$ & $4,03 \mathrm{E}-01$ & $-2,596$ & 0,00944 \\
\hline & ne & $-1,14 \mathrm{E}-01$ & $7,73 \mathrm{E}-02$ & $-1,48 \mathrm{E}+00$ & $1,40 \mathrm{E}-01$ \\
\hline & $e$ & $-1,69 \mathrm{E}-02$ & $1,22 \mathrm{E}-02$ & $-1,38 \mathrm{E}+00$ & $1,67 \mathrm{E}-01$ \\
\hline & $c$ & $-2,30 \mathrm{E}-01$ & $4,18 \mathrm{E}-01$ & $-5,52 \mathrm{E}-01$ & $5,81 \mathrm{E}-01$ \\
\hline & $f a$ & $-9,00 \mathrm{E}-03$ & $1,32 \mathrm{E}-01$ & $-0,068$ & 0,94565 \\
\hline & re & $-9,64 \mathrm{E}-01$ & $3,81 \mathrm{E}-01$ & $-2,533$ & 0,01132 \\
\hline \multirow{13}{*}{ PSOE } & en & $-2,54 \mathrm{E}-01$ & 0,22575 & $-1,124$ & 0,26094 \\
\hline & $r v g$ & $-5,38 \mathrm{E}+00$ & 0,28735 & 18,712 & 0,00001 \\
\hline & b & $6,23 \mathrm{E}-07$ & $1,82 \mathrm{E}-07$ & 3,428 & 0,00061 \\
\hline & $d e$ & $-2,38 \mathrm{E}-02$ & $3,44 \mathrm{E}-01$ & $-0,069$ & 0,94481 \\
\hline & pe & $-2,31 \mathrm{E}-01$ & $4,47 \mathrm{E}-01$ & $-0,517$ & 0,60507 \\
\hline & $s$ & $5,56 \mathrm{E}-02$ & $2,18 \mathrm{E}-01$ & 0,254 & 0,79929 \\
\hline & $n a$ & $-1,05 \mathrm{E}+00$ & $2,29 \mathrm{E}-01$ & $-4,565$ & 0,00001 \\
\hline & as & $4,20 \mathrm{E}-01$ & - & - & - \\
\hline & ne & $-1,04 \mathrm{E}-01$ & $5,52 \mathrm{E}-02$ & $-1,89 \mathrm{E}+00$ & $5,92 \mathrm{E}-02$ \\
\hline & $e$ & $5,78 \mathrm{E}-03$ & $7,68 \mathrm{E}-03$ & $7,53 \mathrm{E}-01$ & $4,52 \mathrm{E}-01$ \\
\hline & $c$ & $6,87 \mathrm{E}-01$ & $2,69 \mathrm{E}-01$ & $2,55 \mathrm{E}+00$ & $1,08 \mathrm{E}-02$ \\
\hline & $f a$ & $1,08 \mathrm{E}-01$ & $7,39 \mathrm{E}-02$ & 1,457 & 0,14500 \\
\hline & re & $2,94 \mathrm{E}-01$ & $2,84 \mathrm{E}-01$ & 1,036 & 0,30018 \\
\hline \multirow{13}{*}{$C D S$} & en & $-2,38 \mathrm{E}-01$ & $5,09 \mathrm{E}-01$ & $-0,467$ & 0,64074 \\
\hline & $r v g$ & $-2,64 \mathrm{E}+00$ & $4,25 \mathrm{E}-01$ & $-6,207$ & 0,00001 \\
\hline & b & $1,91 \mathrm{E}-07$ & $4,04 \mathrm{E}-07$ & 0,473 & 0,63621 \\
\hline & $d e$ & $4,99 \mathrm{E}-01$ & $8,33 \mathrm{E}-01$ & 0,6 & 0,54872 \\
\hline & pe & $-2,09 \mathrm{E}-01$ & $1,11 \mathrm{E}+00$ & $-0,189$ & 0,85030 \\
\hline & $s$ & $-1,29 \mathrm{E}-01$ & $4,66 \mathrm{E}-01$ & $-0,276$ & 0,78233 \\
\hline & $n a$ & $-5,57 \mathrm{E}-01$ & $6,02 \mathrm{E}-01$ & $-0,925$ & 0,35503 \\
\hline & as & $-1,10 \mathrm{E}+00$ & $2,35 \mathrm{E}-01$ & $-4,672$ & 0,00001 \\
\hline & ne & $-1,08 \mathrm{E}-02$ & $9,77 \mathrm{E}-02$ & $-0,111$ & 0,91164 \\
\hline & $e$ & $4,19 \mathrm{E}-03$ & $1,34 \mathrm{E}-02$ & 0,313 & 0,75416 \\
\hline & $c$ & $-1,56 \mathrm{E}-02$ & $5,87 \mathrm{E}-01$ & $-0,027$ & 0,97885 \\
\hline & $f a$ & $-2,47 \mathrm{E}-01$ & $1,17 \mathrm{E}-01$ & $-2,107$ & 0,03516 \\
\hline & re & $-2,38 \mathrm{E}-01$ & $4,89 \mathrm{E}-01$ & $-0,488$ & 0,62588 \\
\hline
\end{tabular}




\section{CUADRO 1 (continuación)}

Estimación de la función de voto en las elecciones generales de 1982

\begin{tabular}{|c|c|c|c|c|c|}
\hline Opción electoral & Variable & Coeficiente & $\begin{array}{l}\text { Desviación } \\
\text { estándar } \\
\text { coeficiente }\end{array}$ & T-Student & $\begin{array}{c}\text { Nivel } \\
\text { significación } \\
{\left[\text { Prob }|t|>x\left({ }^{*}\right)\right]}\end{array}$ \\
\hline \multirow{13}{*}{$U C D$} & $e n$ & $-2,34 \mathrm{E}-01$ & $3,32 \mathrm{E}-01$ & $-0,704$ & 0,48173 \\
\hline & $r v g$ & $-3,48 \mathrm{E}+00$ & $3,91 \mathrm{E}-01$ & $-8,909$ & 0,00001 \\
\hline & $b^{\circ}$ & $-7,01 \mathrm{E}-07$ & $3,56 \mathrm{E}-07$ & $-1,966$ & 0,04928 \\
\hline & de & $-1,55 \mathrm{E}+00$ & $3,81 \mathrm{E}-01$ & $-4,07 \mathrm{E}+00$ & $5,00 \mathrm{E}-05$ \\
\hline & pe & $-1,38 \mathrm{E}+00$ & $8,75 \mathrm{E}-01$ & $-1,57 \mathrm{E}+00$ & $1,16 \mathrm{E}-01$ \\
\hline & $s$ & $-8,46 \mathrm{E}-01$ & $3,60 \mathrm{E}-01$ & $-2,35 \mathrm{E}+00$ & $1,89 \mathrm{E}-02$ \\
\hline & $n a$ & $-1,31 \mathrm{E}+00$ & $4,48 \mathrm{E}-01$ & $-2,929$ & 0,00340 \\
\hline & as & $-5,68 \mathrm{E}-01$ & - & - & - \\
\hline & ne & $6,55 \mathrm{E}-02$ & $9,20 \mathrm{E}-02$ & 0,712 & 0,47642 \\
\hline & $e$ & $3,19 \mathrm{E}-02$ & $1,14 \mathrm{E}-02$ & 2,801 & 0,00510 \\
\hline & $c$ & $-2,93 \mathrm{E}-01$ & $3,86 \mathrm{E}-01$ & $-0,76$ & 0,44719 \\
\hline & $f a$ & $-3,03 \mathrm{E}-01$ & $1,18 \mathrm{E}-01$ & $-2,574$ & 0,01007 \\
\hline & re & $4,75 \mathrm{E}-01$ & $4,17 \mathrm{E}-01$ & 1,139 & 0,25478 \\
\hline \multirow{13}{*}{$\mathrm{CiU}$} & en & $-2,15 \mathrm{E}-01$ & $3,30 \mathrm{E}-01$ & $-0,653$ & 0,51398 \\
\hline & $r v g$ & $-3,36 \mathrm{E}+00$ & $4,46 \mathrm{E}-01$ & $-7,532$ & 0,00001 \\
\hline & $b^{\circ}$ & $4,15 \mathrm{E}-07$ & $2,80 \mathrm{E}-07$ & 1,482 & 0,13842 \\
\hline & de & $-7,37 \mathrm{E}-01$ & 0,37051 & $-1,988$ & 0,04676 \\
\hline & pe & $6,83 \mathrm{E}-01$ & 0,80844 & 0,845 & 0,39815 \\
\hline & $s$ & $-4,87 \mathrm{E}-01$ & $3,48 \mathrm{E}-01$ & $-1,4$ & 0,16163 \\
\hline & na & $1,70 \mathrm{E}+00$ & $2,64 \mathrm{E}-01$ & 6,438 & 0,00001 \\
\hline & as & $-1,48 \mathrm{E}+00$ & - & - & - \\
\hline & ne & $4,31 \mathrm{E}-02$ & 7,64E-02 & 0,564 & 0,57294 \\
\hline & $e$ & $-1,34 \mathrm{E}-02$ & $1,22 \mathrm{E}-02$ & $-1,097$ & 0,27286 \\
\hline & $c$ & $7,57 \mathrm{E}-02$ & $4,03 \mathrm{E}-01$ & 0,188 & 0,85094 \\
\hline & $f a$ & $-4,50 \mathrm{E}-01$ & $1,24 \mathrm{E}-01$ & $-3,618$ & 0,00030 \\
\hline & re & $6,55 \mathrm{E}-01$ & $3,80 \mathrm{E}-01$ & $1,73 \mathrm{E}+00$ & $8,43 \mathrm{E}-02$ \\
\hline \multirow{13}{*}{$A P\left({ }^{* *}\right)$} & en & $-1,48 \mathrm{E}-01$ & $2,68 \mathrm{E}-01$ & $-0,551$ & 0,58140 \\
\hline & rvg & $-4,14 \mathrm{E}+00$ & $3,31 \mathrm{E}-01$ & 12,523 & 0,00001 \\
\hline & $b$ & $2,20 \mathrm{E}-07$ & $2,17 \mathrm{E}-07$ & 1,014 & 0,31068 \\
\hline & de & $-3,86 \mathrm{E}-01$ & $4,82 \mathrm{E}-01$ & $-0,801$ & 0,42337 \\
\hline & pe & $-3,45 \mathrm{E}-01$ & $6,07 \mathrm{E}-01$ & $-0,568$ & 0,56990 \\
\hline & $s$ & $-2,22 \mathrm{E}-01$ & $2,52 \mathrm{E}-01$ & $-0,883$ & 0,37734 \\
\hline & $n a$ & $-1,78 \mathrm{E}+00$ & $3,38 \mathrm{E}-01$ & $-5,26 \mathrm{E}+00$ & $1,00 \mathrm{E}-05$ \\
\hline & as & $-1,15 \mathrm{E}+00$ & - & - & - \\
\hline & ne & $6,36 \mathrm{E}-02$ & $6,37 \mathrm{E}-02$ & $9,97 \mathrm{E}-01$ & $3,19 \mathrm{E}-01$ \\
\hline & $e$ & $1,43 \mathrm{E}-02$ & $9,39 \mathrm{E}-03$ & 1,526 & 0,12693 \\
\hline & $c$ & $4,96 \mathrm{E}-01$ & $3,02 \mathrm{E}-01$ & 1,644 & 0,10026 \\
\hline & $f a$ & $-5,50 \mathrm{E}-02$ & $8,31 \mathrm{E}-02$ & $-0,662$ & 0,50796 \\
\hline & re & $6,10 \mathrm{E}-01$ & $2,97 \mathrm{E}-01$ & 2,053 & 0,04004 \\
\hline
\end{tabular}

$\left(^{*}\right)$ Los niveles de significación utilizados son los de la $\mathrm{N}(0,1)$.

${ }^{(*)}$ Alianza Popular se refundará en 1990. A partir de ese momento pasa a denominarse Partido Popular (PP).

Número observaciones $=812$. Grados libertad $=72$.

Logaritmo de verosimilitud $=-918,5618$. Número iteraciones $=35$.

Ji-Cuadrado $(78$ grados libertad $)=481,4488$. Nivel de significación Ji-Cuadrado $=0,00000001$.

FUENTE: Elaboración propia. 


\section{CUADRO 2}

Estimación de la función de voto en las elecciones generales de 1986

\begin{tabular}{|c|c|c|c|c|c|}
\hline Opción electoral & Variable & Coeficiente & $\begin{array}{l}\text { Desviación } \\
\text { estándar } \\
\text { coeficiente }\end{array}$ & T-Student & $\begin{array}{c}\text { Nivel } \\
\text { significación } \\
{\left[\text { Prob }|t|>x\left(^{*}\right)\right]}\end{array}$ \\
\hline \multirow{21}{*}{$P C E$} & en & $7,43 \mathrm{E}-01$ & 1,5057 & 0,494 & 0,62154 \\
\hline & $c$ & $-5,88 \mathrm{E}-02$ & 0,27256 & $-0,216$ & 0,82906 \\
\hline & $l$ & $-2,18 \mathrm{E}-01$ & $1,35 \mathrm{E}-01$ & $-1,619$ & 0,10555 \\
\hline & $d i$ & $1,28 \mathrm{E}+00$ & $2,29 \mathrm{E}-01$ & 5,58 & 0,00001 \\
\hline & $i$ & $3,30 \mathrm{E}-01$ & $3,85 \mathrm{E}-01$ & 0,856 & 0,39175 \\
\hline & $r v g$ & $-4,13 E+00$ & $6,14 \mathrm{E}-01$ & $-6,725$ & 0,00001 \\
\hline & $v p$ & $6,65 \mathrm{E}+00$ & $6,27 \mathrm{E}-01$ & 10,609 & 0,00001 \\
\hline & $y$ & $6,41 \mathrm{E}-06$ & $1,88 \mathrm{E}-06$ & 3,405 & 0,00066 \\
\hline & $b$ & $-3,36 \mathrm{E}-07$ & - & - & - \\
\hline & de & $1,26 \mathrm{E}+00$ & - & - & - \\
\hline & $p$ & $-8,00 \mathrm{E}-01$ & - & - & - \\
\hline & $\begin{array}{l}P \\
s\end{array}$ & $3,67 \mathrm{E}-01$ & $5,22 \mathrm{E}-01$ & 0,703 & 0,48225 \\
\hline & na & $2,53 \mathrm{E}+00$ & $1,20 \mathrm{E}+00$ & 2,104 & 0,03537 \\
\hline & as & $4,22 \mathrm{E}-01$ & $7,09 \mathrm{E}-01$ & 0,595 & 0,55183 \\
\hline & cs & $-1,08 \mathrm{E}+00$ & $2,48 \mathrm{E}-01$ & $-4,348$ & 0,00001 \\
\hline & ne & $3,80 \mathrm{E}-02$ & $9,08 \mathrm{E}-02$ & 0,418 & 0,67562 \\
\hline & $e$ & $-4,94 \mathrm{E}-02$ & $2,21 \mathrm{E}-02$ & $-2,231$ & 0,02566 \\
\hline & $c i$ & $1,51 \mathrm{E}-01$ & $5,05 \mathrm{E}-01$ & 0,299 & 0,76461 \\
\hline & $f a$ & $4,02 \mathrm{E}-03$ & $1,14 \mathrm{E}-03$ & 3,533 & 0,00041 \\
\hline & re & $1,45 \mathrm{E}+00$ & $6,11 \mathrm{E}-01$ & 2,37 & 0,01777 \\
\hline & pre & $9,49 \mathrm{E}-01$ & $2,20 \mathrm{E}-01$ & 4,312 & 0,00002 \\
\hline \multirow{21}{*}{ PSOE } & en & $3,72 \mathrm{E}-01$ & 1,5395 & 0,242 & 0,80887 \\
\hline & $c$ & $2,31 \mathrm{E}-02$ & 0,22914 & 0,101 & 0,91985 \\
\hline & $l$ & $3,37 \mathrm{E}-01$ & $1,12 \mathrm{E}-01$ & 2,994 & 0,00275 \\
\hline & $d i$ & $5,80 \mathrm{E}-01$ & $1,91 \mathrm{E}-01$ & 3,043 & 0,00234 \\
\hline & $i$ & $-5,38 \mathrm{E}-01$ & $2,72 \mathrm{E}-01$ & $-1,976$ & 0,04816 \\
\hline & $r v g$ & $-2,21 \mathrm{E}+00$ & $5,61 \mathrm{E}-01$ & $-3,934$ & 0,00008 \\
\hline & $v p$ & $6,40 \mathrm{E}+00$ & $3,97 \mathrm{E}-01$ & 16,11 & 0,00001 \\
\hline & $y$ & $2,35 \mathrm{E}-06$ & - & - & - \\
\hline & $h$ & $-3,39 \mathrm{E}-07$ & - & - & - \\
\hline & $d e$ & $9,10 \mathrm{E}-01$ & - & - & - \\
\hline & $p$ & $-3,85 \mathrm{E}-01$ & - & - & - \\
\hline & $s$ & $4,79 \mathrm{E}-01$ & $4,58 \mathrm{E}-01$ & 1,044 & 0,29634 \\
\hline & na & $2,83 \mathrm{E}+00$ & $1,15 \mathrm{E}+00$ & 2,456 & 0,01405 \\
\hline & as & $4,52 \mathrm{E}-01$ & $5,59 \mathrm{E}-01$ & 0,809 & 0,41865 \\
\hline & $c s$ & $-6,29 \mathrm{E}-01$ & $1,99 \mathrm{E}-01$ & $-3,162$ & 0,00157 \\
\hline & ne & $-4,46 \mathrm{E}-02$ & $6,91 \mathrm{E}-02$ & $-0,645$ & 0,51898 \\
\hline & $e$ & $-5,48 \mathrm{E}-02$ & $1,84 \mathrm{E}-02$ & $-2,97$ & 0,00298 \\
\hline & $c i$ & $9,92 \mathrm{E}-01$ & $2,40 \mathrm{E}-01$ & 4,137 & 0,00004 \\
\hline & $f a$ & $3,58 \mathrm{E}-03$ & $5,55 \mathrm{E}-04$ & 6,463 & 0,00001 \\
\hline & re & $2,77 E+00$ & $5,75 \mathrm{E}-01$ & 4,824 & 0,00001 \\
\hline & pre & $4,85 \mathrm{E}-01$ & $1,54 \mathrm{E}-01$ & 3,152 & 0,00162 \\
\hline
\end{tabular}




\section{CUADRO 2 (continuación)}

Estimación de la función de voto en las elecciones generales de 1986

\begin{tabular}{|c|c|c|c|c|c|}
\hline Opción electoral & Variable & Coeficiente & $\begin{array}{l}\text { Desviación } \\
\text { estándar } \\
\text { coeficiente }\end{array}$ & T-Student & $\begin{array}{c}\text { Nivel } \\
\text { significación } \\
{\left[\text { Prob }|t|>x\left({ }^{*}\right)\right]}\end{array}$ \\
\hline \multirow{21}{*}{$C D S$} & en & $1,20 \mathrm{E}+00$ & 1,5343 & 0,784 & 0,4331 \\
\hline & $c$ & $-1,60 \mathrm{E}-01$ & 0,24688 & $-0,65$ & 0,51587 \\
\hline & $l$ & $3,21 \mathrm{E}-01$ & $1,30 \mathrm{E}-01$ & 2,461 & 0,01386 \\
\hline & $d i$ & $3,93 \mathrm{E}-01$ & $2,16 \mathrm{E}-01$ & 1,817 & 0,06923 \\
\hline & $i$ & $-2,94 \mathrm{E}-01$ & $3,10 \mathrm{E}-01$ & $-0,949$ & 0,34265 \\
\hline & $r v g$ & $-5,40 \mathrm{E}+00$ & $5,95 \mathrm{E}-01$ & $-9,076$ & 0,00001 \\
\hline & $v p$ & $6,56 \mathrm{E}+00$ & $5,84 \mathrm{E}-01$ & 11,239 & 0,00001 \\
\hline & $y$ & $3,39 \mathrm{E}-06$ & $1,84 \mathrm{E}-06$ & 1,848 & 0,06467 \\
\hline & $h$ & $-3,59 \mathrm{E}-07$ & - & - & - \\
\hline & de & $4,12 \mathrm{E}-01$ & - & - & - \\
\hline & $p$ & $-7,36 \mathrm{E}-01$ & - & - & - \\
\hline & s & $2,74 \mathrm{E}-01$ & $4,88 \mathrm{E}-01$ & 0,562 & 0,57427 \\
\hline & $n a$ & $2,21 \mathrm{E}+00$ & $1,22 \mathrm{E}+00$ & 1,819 & 0,06891 \\
\hline & as & $6,29 \mathrm{E}-01$ & $6,34 \mathrm{E}-01$ & 0,992 & 0,32103 \\
\hline & $c s$ & $-4,39 \mathrm{E}-01$ & $2,26 \mathrm{E}-01$ & $-1,945$ & 0,05173 \\
\hline & ne & $9,41 \mathrm{E}-02$ & $8,72 \mathrm{E}-02$ & 1,079 & 0,28080 \\
\hline & $e$ & $-3,32 \mathrm{E}-02$ & $1,92 \mathrm{E}-02$ & $-1,733$ & 0,08304 \\
\hline & $c i$ & $4,25 \mathrm{E}-01$ & $3,67 \mathrm{E}-01$ & 1,158 & 0,24677 \\
\hline & $f a$ & 2,89E-03 & $1,36 \mathrm{E}-03$ & 2,117 & 0,03429 \\
\hline & re & $3,08 \mathrm{E}+00$ & $6,64 \mathrm{E}-01$ & 4,647 & 0,00001 \\
\hline & pre & $1,58 \mathrm{E}-01$ & $1,74 \mathrm{E}-01$ & 0,909 & 0,36343 \\
\hline \multirow{21}{*}{$\mathrm{CiU}$} & en & $-1,67 \mathrm{E}+00$ & 1,6142 & $-1,033$ & 0,30157 \\
\hline & $c$ & $-2,61 \mathrm{E}-01$ & 0,2809 & $-0,927$ & 0,3537 \\
\hline & $l$ & $-1,34 \mathrm{E}-01$ & $1,39 \mathrm{E}-01$ & $-0,962$ & 0,33604 \\
\hline & $d i$ & $5,76 \mathrm{E}-01$ & $2,23 \mathrm{E}-01$ & 2,582 & 0,00983 \\
\hline & $i$ & $-6,06 \mathrm{E}-01$ & $3,25 \mathrm{E}-01$ & $-1,864$ & 0,06226 \\
\hline & $r v g$ & $-3,33 \mathrm{E}+00$ & $7,11 \mathrm{E}-01$ & $-4,687$ & 0,00001 \\
\hline & $v p$ & $6,61 \mathrm{E}+00$ & $7,72 \mathrm{E}-01$ & 8,567 & 0,00001 \\
\hline & $y$ & $8,50 \mathrm{E}-06$ & $5,42 \mathrm{E}-06$ & 1,567 & 0,11706 \\
\hline & h & $-8,63 \mathrm{E}-07$ & $2,12 \mathrm{E}-07$ & $-4,067$ & 0,00005 \\
\hline & $d e$ & $-1,13 \mathrm{E}+00$ & - & - & - \\
\hline & $p$ & $2,06 \mathrm{E}+00$ & - & - & - \\
\hline & s & $1,44 \mathrm{E}-01$ & $6,03 \mathrm{E}-01$ & 0,238 & 0,81164 \\
\hline & $n a$ & $9,09 \mathrm{E}+00$ & $1,22 \mathrm{E}+00$ & 7,422 & 0,00001 \\
\hline & as & $-8,19 \mathrm{E}-01$ & $6,70 \mathrm{E}-01$ & $-1,222$ & 0,22169 \\
\hline & $c s$ & $-1,77 \mathrm{E}-01$ & $2,72 \mathrm{E}-01$ & $-0,649$ & 0,51651 \\
\hline & ne & $1,51 \mathrm{E}-01$ & $1,52 \mathrm{E}-01$ & 0,992 & 0,32138 \\
\hline & $e$ & $-1,18 \mathrm{E}-02$ & $2,18 \mathrm{E}-02$ & $-0,544$ & 0,58639 \\
\hline & $c i$ & $-8,32 \mathrm{E}-02$ & $6,93 \mathrm{E}-01$ & $-0,12$ & 0,90441 \\
\hline & $f a$ & $6,20 \mathrm{E}-03$ & $1,65 \mathrm{E}-03$ & 3,752 & 0,00018 \\
\hline & re & $2,10 \mathrm{E}+00$ & $6,16 \mathrm{E}-01$ & 3,404 & 0,00066 \\
\hline & pre & $1,43 \mathrm{E}-01$ & $2,01 \mathrm{E}-01$ & 0,714 & 0,47530 \\
\hline
\end{tabular}




\section{CUADRO 2 (continuación)}

Estimación de la función de voto en las elecciones generales de 1986

\begin{tabular}{|c|c|c|c|c|c|}
\hline Opción electoral & Variable & Coeficiente & $\begin{array}{l}\text { Desviación } \\
\text { estándar } \\
\text { coeficiente }\end{array}$ & T-Student & $\begin{array}{c}\text { Nivel } \\
\text { significación } \\
{\left[\text { Prob }|t|>x\left(^{*}\right)\right]}\end{array}$ \\
\hline \multirow{21}{*}{$C P\left({ }^{* *}\right)$} & en & $-1,07 \mathrm{E}+00$ & 1,612 & $-0,663$ & 0,50735 \\
\hline & $c$ & $-1,75 \mathrm{E}-01$ & 0,2563 & $-0,683$ & 0,49439 \\
\hline & $l$ & $4,93 \mathrm{E}-01$ & $1,38 \mathrm{E}-01$ & 3,584 & 0,00034 \\
\hline & $d i$ & $-1,63 \mathrm{E}-03$ & $2,08 \mathrm{E}-01$ & $-0,008$ & 0,99376 \\
\hline & $i$ & $4,29 \mathrm{E}-01$ & $3,24 \mathrm{E}-01$ & 1,324 & 0,18550 \\
\hline & $r v g$ & $-8,74 \mathrm{E}+00$ & $4,30 \mathrm{E}-01$ & 20,329 & 0,00001 \\
\hline & $v p$ & $-5,95 \mathrm{E}-01$ & $5,67 \mathrm{E}-01$ & $-1,049$ & 0,29405 \\
\hline & $y$ & $8,49 \mathrm{E}-06$ & $2,62 \mathrm{E}-06$ & 3,24 & 0,00119 \\
\hline & $b$ & $-1,32 \mathrm{E}-07$ & - & - & - \\
\hline & $d e$ & $1,50 \mathrm{E}+00$ & - & - & - \\
\hline & $p$ & $-1,19 \mathrm{E}+00$ & - & - & - \\
\hline & $s$ & $2,26 \mathrm{E}-01$ & $5,35 \mathrm{E}-01$ & 0,423 & 0,67251 \\
\hline & na & $1,20 \mathrm{E}+00$ & $1,19 \mathrm{E}+00$ & 1,006 & 0,31426 \\
\hline & as & $-1,23 \mathrm{E}+00$ & $5,81 \mathrm{E}-01$ & $-2,11$ & 0,03486 \\
\hline & $c s$ & $-2,58 \mathrm{E}-01$ & $2,57 \mathrm{E}-01$ & $-1,004$ & 0,31526 \\
\hline & ne & $1,21 \mathrm{E}-01$ & $1,08 \mathrm{E}-01$ & 1,128 & 0,25931 \\
\hline & $e$ & $-4,46 \mathrm{E}-02$ & $2,09 \mathrm{E}-02$ & $-2,136$ & 0,03266 \\
\hline & $c i$ & $9,34 \mathrm{E}-01$ & $6,28 \mathrm{E}-01$ & 1,487 & 0,13702 \\
\hline & $f a$ & 1,07E-03 & $1,05 \mathrm{E}-03$ & 1,025 & 0,30538 \\
\hline & re & $2,54 \mathrm{E}+00$ & $1,16 \mathrm{E}+00$ & 2,192 & 0,02839 \\
\hline & pre & $-1,04 \mathrm{E}-01$ & $1,79 \mathrm{E}-01$ & $-0,583$ & 0,55989 \\
\hline
\end{tabular}

(*) Los niveles de significación utilizados son los de la $\mathrm{N}(0,1)$.

(**) Coalición Popular (CP) estaba formada por AP, PDP y UL. En 1990 se refunda el Partido Popular (PP).

Número observaciones $=1.096$. Grados libertad $=100$.

Logaritmo de verosimilitud $=-538,0610$. Número iteraciones $=5$.

Ji-Cuadrado $(72$ grados libertad $)=481,4488$. Nivel de significación Ji-Cuadrado $=0,00000001$.

FUENTE: Elaboración propia. 


\section{CUADRO 3}

Estimación de la función de voto en las elecciones generales de 1989

\begin{tabular}{|c|c|c|c|c|c|}
\hline Opción electoral & Variable & Coeficiente & $\begin{array}{l}\text { Desviación } \\
\text { estándar } \\
\text { coeficiente }\end{array}$ & T-Student & $\begin{array}{c}\text { Nivel } \\
\text { significación } \\
{\left[\text { Prob }|t|>x\left({ }^{*}\right)\right]}\end{array}$ \\
\hline \multirow{14}{*}{$I U$} & en & $1,75 \mathrm{E}-01$ & - & - & - \\
\hline & $c$ & $-6,36 \mathrm{E}-02$ & 0,36621 & $-0,174$ & 0,86202 \\
\hline & $l$ & $-3,42 \mathrm{E}-02$ & $1,92 \mathrm{E}-01$ & $-0,178$ & 0,85876 \\
\hline & $d i$ & $2,98 \mathrm{E}-01$ & $8,08 \mathrm{E}-01$ & 3,693 & 0,00022 \\
\hline & $i$ & $3,99 \mathrm{E}-01$ & $2,56 \mathrm{E}-01$ & 1,555 & 0,12001 \\
\hline & $r v g$ & $4,41 \mathrm{E}-01$ & $8,26 \mathrm{E}-01$ & 0,534 & 0,59358 \\
\hline & b & $8,01 \mathrm{E}-07$ & $4,67 \mathrm{E}-01$ & 1,714 & 0,08646 \\
\hline & $d e$ & $3,08 \mathrm{E}-01$ & - & - & - \\
\hline & na & $-1,61 E+00$ & $3,68 \mathrm{E}-01$ & $-4,38 \mathrm{E}+00$ & $1,00 \mathrm{E}-05$ \\
\hline & as & $1,13 \mathrm{E}-01$ & $2,62 \mathrm{E}-01$ & $4,32 \mathrm{E}-01$ & $6,65 \mathrm{E}-01$ \\
\hline & ne & $-1,00 \mathrm{E}-01$ & - & - & - \\
\hline & $e$ & $-5,78 \mathrm{E}-02$ & - & - & - \\
\hline & $c$ & $3,59 \mathrm{E}-01$ & - & - & - \\
\hline & re & $-1,81 \mathrm{E}+00$ & $3,84 \mathrm{E}-01$ & $-4,716$ & 0,00001 \\
\hline \multirow{14}{*}{ PSOE } & en & $-5,38 \mathrm{E}-01$ & - & - & - \\
\hline & $c$ & $1,36 \mathrm{E}-02$ & 0,3304 & 0,041 & 0,96707 \\
\hline & $l$ & $-8,47 \mathrm{E}-02$ & $1,71 \mathrm{E}-01$ & $-0,495$ & 0,62069 \\
\hline & $d i$ & $-5,40 \mathrm{E}-02$ & - & - & - \\
\hline & $i$ & $-1,78 \mathrm{E}-01$ & $1,26 \mathrm{E}-01$ & $-1,409$ & 0,15880 \\
\hline & $r v g$ & $1,75 \mathrm{E}+00$ & $7,38 \mathrm{E}-01$ & 2,363 & 0,01811 \\
\hline & $b$ & $5,45 \mathrm{E}-07$ & $4,39 \mathrm{E}-01$ & 1,242 & 0,21436 \\
\hline & de & $3,02 \mathrm{E}-01$ & - & - & - \\
\hline & na & $-1,64 \mathrm{E}+00$ & $2,26 \mathrm{E}-01$ & $-7,25 E+00$ & $1,00 \mathrm{E}-05$ \\
\hline & as & $3,86 \mathrm{E}-02$ & - & - & - \\
\hline & ne & $-2,01 \mathrm{E}-01$ & - & - & - \\
\hline & $e$ & $-2,78 \mathrm{E}-02$ & - & - & - \\
\hline & $c$ & $6,95 \mathrm{E}-02$ & - & - & - \\
\hline & re & $-2,48 \mathrm{E}-01$ & $3,78 \mathrm{E}-01$ & $-0,656$ & 0,51153 \\
\hline \multirow{14}{*}{$C D S$} & en & $-8,91 \mathrm{E}-01$ & - & - & - \\
\hline & $c$ & $-1,69 \mathrm{E}-01$ & $3,56 \mathrm{E}-01$ & $-0,474$ & 0,63582 \\
\hline & $l$ & $-2,06 \mathrm{E}-01$ & $1,98 \mathrm{E}-01$ & $-1,04$ & 0,29821 \\
\hline & $d i$ & $-2,13 \mathrm{E}-01$ & - & - & - \\
\hline & $i$ & $-5,41 \mathrm{E}-01$ & 0,18715 & $-2,891$ & 0,00384 \\
\hline & $r v g$ & $1,67 \mathrm{E}+00$ & 0,77593 & 2,149 & 0,03166 \\
\hline & b & $1,14 \mathrm{E}-06$ & $5,23 \mathrm{E}-01$ & 2,168 & 0,03013 \\
\hline & $d e$ & $-7,47 \mathrm{E}-01$ & - & - & - \\
\hline & na & $-2,59 \mathrm{E}+00$ & $4,72 \mathrm{E}-01$ & $-5,498$ & 0,00001 \\
\hline & as & $-4,56 \mathrm{E}-01$ & $2,96 \mathrm{E}-01$ & $-1,542$ & 0,12299 \\
\hline & ne & $4,95 \mathrm{E}-02$ & - & - & - \\
\hline & $e$ & $9,55 \mathrm{E}-03$ & - & - & - \\
\hline & $c$ & $-5,52 \mathrm{E}-01$ & - & - & - \\
\hline & re & $5,50 \mathrm{E}-01$ & $6,79 \mathrm{E}-01$ & $8,11 \mathrm{E}-01$ & $4,18 \mathrm{E}-01$ \\
\hline
\end{tabular}




\section{CUADRO 3 (continuación)}

Estimación de la función de voto en las elecciones generales de 1989

\begin{tabular}{|c|c|c|c|c|c|}
\hline Opción electoral & Variable & Coeficiente & $\begin{array}{c}\text { Desviación } \\
\text { estándar } \\
\text { coeficiente }\end{array}$ & T-Student & $\begin{array}{c}\text { Nivel } \\
\text { significación } \\
{\left[\text { Prob }|t|>x\left(^{*}\right)\right]}\end{array}$ \\
\hline \multirow{14}{*}{$\mathrm{CiU}$} & en & $-1,41 \mathrm{E}-01$ & - & - & - \\
\hline & $c$ & $-1,87 \mathrm{E}-01$ & $3,57 \mathrm{E}-01$ & $-0,524$ & 0,60041 \\
\hline & $l$ & $-7,72 \mathrm{E}-02$ & $1,98 \mathrm{E}-01$ & $-0,389$ & 0,69698 \\
\hline & $d i$ & $-1,97 \mathrm{E}-01$ & - & - & - \\
\hline & $i$ & $1,82 \mathrm{E}-01$ & $1,96 \mathrm{E}-01$ & 0,929 & 0,35309 \\
\hline & $r v g$ & $6,60 \mathrm{E}-01$ & $7,42 \mathrm{E}-01$ & 0,889 & 0,37382 \\
\hline & $b^{\circ}$ & $1,64 \mathrm{E}-07$ & $5,38 \mathrm{E}-01$ & 0,305 & 0,76072 \\
\hline & de & $1,51 \mathrm{E}-01$ & - & - & - \\
\hline & $n a$ & $1,82 \mathrm{E}+00$ & 0,28167 & 6,466 & 0,00001 \\
\hline & as & $-1,47 \mathrm{E}+00$ & 0,28129 & $-5,227$ & 0,00001 \\
\hline & ne & $-1,39 \mathrm{E}-01$ & - & - & - \\
\hline & $e$ & $-1,01 \mathrm{E}-02$ & - & - & - \\
\hline & $c$ & $-4,44 \mathrm{E}-01$ & - & - & - \\
\hline & re & $5,97 \mathrm{E}-01$ & $5,21 \mathrm{E}-01$ & 1,145 & 0,25223 \\
\hline \multirow{14}{*}{$P P$} & en & $1,48 \mathrm{E}-01$ & - & - & - \\
\hline & $c$ & $-2,64 \mathrm{E}-01$ & $3,20 \mathrm{E}-01$ & $-0,828$ & 0,40795 \\
\hline & $l$ & $1,20 \mathrm{E}-01$ & $1,31 \mathrm{E}-01$ & $9,14 \mathrm{E}-01$ & $3,61 \mathrm{E}-01$ \\
\hline & $d i$ & $-3,46 \mathrm{E}-01$ & - & - & - \\
\hline & $i$ & $-2,71 \mathrm{E}-02$ & $2,22 \mathrm{E}-01$ & $-1,22 \mathrm{E}-01$ & $9,03 \mathrm{E}-01$ \\
\hline & $r v g$ & $-3,74 \mathrm{E}+00$ & $6,93 \mathrm{E}-01$ & $-5,399$ & 0,00001 \\
\hline & b & $4,34 \mathrm{E}-07$ & $5,31 \mathrm{E}-01$ & 0,817 & 0,41394 \\
\hline & $d e$ & $1,48 \mathrm{E}-01$ & - & - & - \\
\hline & na & $-2,28 \mathrm{E}+00$ & $2,88 \mathrm{E}-01$ & $-7,913$ & 0,00001 \\
\hline & as & $-6,70 \mathrm{E}-01$ & $1,72 \mathrm{E}-01$ & $-3,885$ & 0,00010 \\
\hline & ne & $2,87 \mathrm{E}-02$ & - & - & - \\
\hline & $e$ & $1,60 \mathrm{E}-02$ & - & - & - \\
\hline & $c$ & $3,58 \mathrm{E}-01$ & - & - & - \\
\hline & re & $9,79 \mathrm{E}-01$ & $6,07 \mathrm{E}-01$ & 1,614 & 0,10652 \\
\hline
\end{tabular}

${ }^{*}$ ) Los niveles de significación utilizados son los de la $\mathrm{N}(0,1)$.

Número observaciones $=670$. Grados libertad $=70$.

Logaritmo de verosimilitud $=-511,8646$. Número iteraciones $=35$.

Ji-Cuadrado $(84$ grados libertad $)=732,8555$. Nivel de significación Ji-Cuadrado $=0,00000001$.

FUENTE: Elaboración propia. 


\section{CUADRO 4}

Estimación de la función de voto en las elecciones generales de 1993

\begin{tabular}{|c|c|c|c|c|c|}
\hline Opción electoral & Variable & Coeficiente & $\begin{array}{l}\text { Desviación } \\
\text { estándar } \\
\text { coeficiente }\end{array}$ & T-Student & $\begin{array}{c}\text { Nivel } \\
\text { significación } \\
{\left[\text { Prob }|t|>x\left({ }^{*}\right)\right]}\end{array}$ \\
\hline \multirow{18}{*}{$I U$} & $t v$ & $2,44 \mathrm{E}-01$ & 0,46914 & 0,521 & 0,60263 \\
\hline & $l$ & $9,03 \mathrm{E}-01$ & 0,14823 & 6,091 & 0,00001 \\
\hline & $d i$ & $-5,49 \mathrm{E}-01$ & $3,99 \mathrm{E}-01$ & $-1,374$ & 0,16942 \\
\hline & $i$ & $-7,89 \mathrm{E}-01$ & $3,07 \mathrm{E}-01$ & $-2,569$ & 0,01020 \\
\hline & $r v g$ & $9,84 \mathrm{E}-02$ & $8,61 \mathrm{E}-01$ & 0,114 & 0,90901 \\
\hline & rva & $-7,48 \mathrm{E}-01$ & $8,58 \mathrm{E}-01$ & $-0,872$ & 0,38318 \\
\hline & pe & $7,95 \mathrm{E}-01$ & $9,46 \mathrm{E}-01$ & 0,84 & 0,40066 \\
\hline & $p p$ & $-8,49 \mathrm{E}-01$ & $7,10 \mathrm{E}-01$ & $-1,197$ & 0,23132 \\
\hline & $b$ & $1,77 \mathrm{E}-06$ & $4,73 \mathrm{E}-07$ & $3,74 \mathrm{E}+00$ & $1,80 \mathrm{E}-04$ \\
\hline & $y$ & $2,76 \mathrm{E}-01$ & $6,87 \mathrm{E}-01$ & $4,02 \mathrm{E}-01$ & $6,87 \mathrm{E}-01$ \\
\hline & de & $-6,77 \mathrm{E}-01$ & $8,38 \mathrm{E}-01$ & $-8,08 \mathrm{E}-01$ & $4,19 \mathrm{E}-01$ \\
\hline & $p$ & $-1,08 \mathrm{E}-02$ & $1,17 \mathrm{E}+00$ & $-0,009$ & 0,99260 \\
\hline & $s$ & $-1,65 \mathrm{E}-01$ & $8,76 \mathrm{E}-01$ & $-0,188$ & 0,85077 \\
\hline & $n a$ & $-4,06 \mathrm{E}+00$ & $8,48 \mathrm{E}-01$ & $-4,79$ & 0,00001 \\
\hline & as & $-1,31 \mathrm{E}+00$ & $4,91 \mathrm{E}-01$ & $-2,667$ & 0,00765 \\
\hline & $s p$ & $8,13 \mathrm{E}-01$ & $5,23 \mathrm{E}-01$ & 1,553 & 0,12038 \\
\hline & ne & $1,25 \mathrm{E}-01$ & $1,54 \mathrm{E}-01$ & 0,814 & 0,41566 \\
\hline & $e$ & $4,08 \mathrm{E}-03$ & $2,69 \mathrm{E}-02$ & 0,152 & 0,87941 \\
\hline \multirow{18}{*}{ PSOE } & $t v$ & $8,98 \mathrm{E}-01$ & 0,4708 & 1,907 & 0,0565 \\
\hline & $l$ & $8,86 \mathrm{E}-01$ & 0,14849 & 5,965 & 0,00001 \\
\hline & $d i$ & $-1,02 \mathrm{E}+00$ & $4,26 \mathrm{E}-01$ & $-2,397$ & 0,01652 \\
\hline & $i$ & $-7,81 \mathrm{E}-01$ & $3,02 \mathrm{E}-01$ & $-2,588$ & 0,00966 \\
\hline & $v r g$ & $1,62 \mathrm{E}+00$ & $8,97 \mathrm{E}-01$ & 1,812 & 0,06999 \\
\hline & vra & $-9,21 \mathrm{E}-01$ & $8,78 \mathrm{E}-01$ & $-1,049$ & 0,29432 \\
\hline & pe & $-5,85 \mathrm{E}-02$ & $9,76 \mathrm{E}-01$ & $-0,06$ & 0,95215 \\
\hline & $p p$ & $-1,13 \mathrm{E}+00$ & $7,08 \mathrm{E}-01$ & $-1,601$ & 0,10942 \\
\hline & b & $1,71 \mathrm{E}-06$ & $4,70 \mathrm{E}-07$ & $3,64 \mathrm{E}+00$ & $2,80 \mathrm{E}-04$ \\
\hline & $y$ & $3,59 \mathrm{E}-01$ & $6,79 \mathrm{E}-01$ & $5,29 \mathrm{E}-01$ & $5,97 \mathrm{E}-01$ \\
\hline & de & $-6,28 \mathrm{E}-01$ & $8,24 \mathrm{E}-01$ & $-7,62 \mathrm{E}-01$ & $4,46 \mathrm{E}-01$ \\
\hline & $p$ & $5,09 \mathrm{E}-01$ & $1,10 \mathrm{E}+00$ & 0,464 & 0,64233 \\
\hline & $s$ & $-4,57 \mathrm{E}-01$ & $8,80 \mathrm{E}-01$ & $-0,52$ & 0,60315 \\
\hline & $n a$ & $-3,30 \mathrm{E}+00$ & $8,62 \mathrm{E}-01$ & $-3,826$ & 0,00013 \\
\hline & as & $-7,75 \mathrm{E}-01$ & $4,99 \mathrm{E}-01$ & $-1,553$ & 0,12030 \\
\hline & $s p$ & $1,08 \mathrm{E}+00$ & $5,26 \mathrm{E}-01$ & 2,056 & 0,03975 \\
\hline & ne & $1,27 \mathrm{E}-01$ & $1,58 \mathrm{E}-01$ & 0,802 & 0,42243 \\
\hline & $e$ & $1,31 \mathrm{E}-02$ & $2,67 \mathrm{E}-02$ & 0,488 & 0,62533 \\
\hline
\end{tabular}




\section{CUADRO 4 (continuación)}

Estimación de la función de voto en las elecciones generales de 1993

\begin{tabular}{|c|c|c|c|c|c|}
\hline Opción electoral & Variable & Coeficiente & $\begin{array}{l}\text { Desviación } \\
\text { estándar } \\
\text { coeficiente }\end{array}$ & T-Student & $\begin{array}{c}\text { Nivel } \\
\text { significación } \\
{\left[\text { Prob }|t|>x\left(^{*}\right)\right]}\end{array}$ \\
\hline \multirow{18}{*}{$\mathrm{CiU}$} & $t v$ & $-1,22 \mathrm{E}+00$ & 0,56557 & $-2,161$ & 0,03073 \\
\hline & $l$ & $6,25 \mathrm{E}-01$ & 0,16228 & 3,849 & 0,00012 \\
\hline & $d i$ & $-7,03 \mathrm{E}-01$ & $3,84 \mathrm{E}-01$ & $-1,831$ & 0,06717 \\
\hline & $i$ & $-3,51 \mathrm{E}-01$ & $3,72 \mathrm{E}-01$ & $-0,942$ & 0,34629 \\
\hline & $v r g$ & $-3,60 \mathrm{E}-01$ & $9,04 \mathrm{E}-01$ & $-0,398$ & 0,69092 \\
\hline & vra & $3,92 \mathrm{E}-01$ & $9,02 \mathrm{E}-01$ & 0,435 & 0,66373 \\
\hline & $p e$ & $-5,94 \mathrm{E}-01$ & $1,08 \mathrm{E}+00$ & $-0,548$ & 0,58363 \\
\hline & $p p$ & $-2,69 \mathrm{E}-01$ & $8,24 \mathrm{E}-01$ & $-0,326$ & 0,74434 \\
\hline & $h$ & $2,12 \mathrm{E}-06$ & $5,04 \mathrm{E}-07$ & $4,21 \mathrm{E}+00$ & $3,00 \mathrm{E}-05$ \\
\hline & $y$ & $1,03 \mathrm{E}+00$ & $7,71 \mathrm{E}-01$ & $1,34 \mathrm{E}+00$ & $1,81 \mathrm{E}-01$ \\
\hline & de & $-9,28 \mathrm{E}-01$ & $9,83 \mathrm{E}-01$ & $-9,45 \mathrm{E}-01$ & $3,45 \mathrm{E}-01$ \\
\hline & $p$ & $3,19 \mathrm{E}-01$ & $1,17 \mathrm{E}+00$ & 0,272 & 0,78548 \\
\hline & $s$ & $-1,07 \mathrm{E}+00$ & $8,87 \mathrm{E}-01$ & $-1,203$ & 0,22886 \\
\hline & na & $-1,08 \mathrm{E}-02$ & $8,28 \mathrm{E}-01$ & $-0,013$ & 0,98954 \\
\hline & as & $5,98 \mathrm{E}-01$ & $5,38 \mathrm{E}-01$ & 1,112 & 0,26631 \\
\hline & $s p$ & $1,43 E+00$ & $5,42 \mathrm{E}-01$ & 2,638 & 0,00834 \\
\hline & ne & $-8,96 \mathrm{E}-02$ & $1,79 \mathrm{E}-01$ & $-0,499$ & 0,61767 \\
\hline & $e$ & $1,35 \mathrm{E}-02$ & $2,89 \mathrm{E}-02$ & 0,468 & 0,63965 \\
\hline \multirow{18}{*}{$P P$} & $t v$ & $2,44 \mathrm{E}-01$ & 0,45925 & 0,53 & 0,5959 \\
\hline & $l$ & $7,51 \mathrm{E}-01$ & 0,15879 & 4,729 & 0,00001 \\
\hline & $d i$ & $1,15 \mathrm{E}+00$ & $3,79 \mathrm{E}-01$ & 3,036 & 0,00240 \\
\hline & $i$ & $-1,36 \mathrm{E}+00$ & $3,38 \mathrm{E}-01$ & $-4,029$ & 0,00006 \\
\hline & $v r g$ & $-4,54 \mathrm{E}-01$ & $8,62 \mathrm{E}-01$ & $-0,527$ & 0,59789 \\
\hline & vra & $-4,97 \mathrm{E}-01$ & $9,21 \mathrm{E}-01$ & $-0,54$ & 0,58943 \\
\hline & pe & $6,30 \mathrm{E}-01$ & $1,04 \mathrm{E}+00$ & 0,604 & 0,54603 \\
\hline & $p p$ & $-1,87 \mathrm{E}-01$ & $8,06 \mathrm{E}-01$ & $-0,232$ & 0,81625 \\
\hline & $h$ & $2,22 \mathrm{E}-06$ & $4,94 \mathrm{E}-07$ & $4,49 \mathrm{E}+00$ & $1,00 \mathrm{E}-05$ \\
\hline & $y$ & $3,38 \mathrm{E}-01$ & $7,27 \mathrm{E}-01$ & $4,65 \mathrm{E}-01$ & $6,42 \mathrm{E}-01$ \\
\hline & de & $-7,90 \mathrm{E}-02$ & $9,62 \mathrm{E}-01$ & $-8,20 \mathrm{E}-02$ & $9,35 \mathrm{E}-01$ \\
\hline & $p$ & $6,13 \mathrm{E}-02$ & $1,17 \mathrm{E}+00$ & 0,053 & 0,95808 \\
\hline & $s$ & $1,33 \mathrm{E}-01$ & $8,73 \mathrm{E}-01$ & 0,152 & 0,87892 \\
\hline & $n a$ & $-4,31 \mathrm{E}+00$ & $8,89 \mathrm{E}-01$ & $-4,847$ & 0,00001 \\
\hline & as & $-9,93 \mathrm{E}-01$ & $5,21 \mathrm{E}-01$ & $-1,907$ & 0,05654 \\
\hline & $s p$ & $1,02 \mathrm{E}+00$ & $5,81 \mathrm{E}-01$ & 1,75 & 0,08020 \\
\hline & ne & $7,49 \mathrm{E}-02$ & $1,56 \mathrm{E}-01$ & 0,48 & 0,63113 \\
\hline & $e$ & $6,36 \mathrm{E}-03$ & $2,75 \mathrm{E}-02$ & 0,231 & 0,81724 \\
\hline
\end{tabular}

${ }^{*}$ ) Los niveles de significación utilizados son los de la $\mathrm{N}(0,1)$.

Número observaciones $=742$. Grados libertad $=68$.

Logaritmo de verosimilitud $=-347,2649$. Número iteraciones $=5$.

Ji-Cuadrado $(73$ grados libertad $)=1144,842$. Nivel de significación Ji-Cuadrado $=0,00000001$.

FUENTE: Elaboración propia. 


\section{CUADRO 5}

Estimación de la función de voto en las elecciones generales de 1996

\begin{tabular}{|c|c|c|c|c|c|}
\hline Opción electoral & Variable & Coeficiente & $\begin{array}{l}\text { Desviación } \\
\text { estándar } \\
\text { coeficiente }\end{array}$ & T-Student & $\begin{array}{c}\text { Nivel } \\
\text { significación } \\
{\left[\text { Prob }|t|>x\left({ }^{*}\right)\right]}\end{array}$ \\
\hline \multirow{16}{*}{$I U$} & $c$ & $6,96 \mathrm{E}-01$ & 0,20527 & 3,389 & 0,0007 \\
\hline & $l$ & $1,52 \mathrm{E}-01$ & $8,06 \mathrm{E}-02$ & 1,88 & 0,06013 \\
\hline & $d i$ & $6,86 \mathrm{E}-01$ & $1,23 \mathrm{E}-01$ & 5,587 & 0,00001 \\
\hline & $c g$ & $2,31 \mathrm{E}+00$ & $4,11 \mathrm{E}-01$ & 5,628 & 0,00001 \\
\hline & $r v g$ & $-8,55 \mathrm{E}-01$ & $3,81 \mathrm{E}-01$ & $-2,243$ & 0,02487 \\
\hline & $r v a$ & $-4,23 \mathrm{E}-01$ & $3,51 \mathrm{E}-01$ & $-1,206$ & 0,22785 \\
\hline & $h$ & $1,85 \mathrm{E}-06$ & $2,83 \mathrm{E}-07$ & 6,533 & 0,00001 \\
\hline & $y$ & $-1,66 \mathrm{E}-02$ & $5,00 \mathrm{E}-01$ & $-0,033$ & 0,97355 \\
\hline & de & $1,39 \mathrm{E}+00$ & $6,78 \mathrm{E}-01$ & 2,055 & 0,03991 \\
\hline & $p$ & $-1,58 \mathrm{E}-01$ & $5,91 \mathrm{E}-01$ & $-2,68 \mathrm{E}-01$ & $7,89 \mathrm{E}-01$ \\
\hline & $s$ & $3,07 \mathrm{E}-01$ & $4,80 \mathrm{E}-01$ & $6,40 \mathrm{E}-01$ & $5,22 \mathrm{E}-01$ \\
\hline & $n a$ & $-3,57 \mathrm{E}+00$ & $2,80 \mathrm{E}-01$ & $-1,27 \mathrm{E}+01$ & $1,00 \mathrm{E}-05$ \\
\hline & as & $-1,62 \mathrm{E}-02$ & $3,87 \mathrm{E}-01$ & $-0,042$ & 0,96662 \\
\hline & $a p$ & $8,80 \mathrm{E}-01$ & $6,00 \mathrm{E}-01$ & 1,467 & 0,14241 \\
\hline & ne & $1,24 \mathrm{E}-01$ & $1,04 \mathrm{E}-01$ & 1,189 & 0,23450 \\
\hline & $e$ & $5,42 \mathrm{E}-03$ & $1,08 \mathrm{E}-03$ & 5,024 & 0,00001 \\
\hline \multirow{16}{*}{ PSOE } & $c$ & $2,47 \mathrm{E}-01$ & 0,2041 & 1,211 & 0,22596 \\
\hline & $l$ & $5,29 \mathrm{E}-01$ & $8,15 \mathrm{E}-02$ & 6,487 & 0,00001 \\
\hline & $d i$ & $2,66 \mathrm{E}-01$ & $1,14 \mathrm{E}-01$ & 2,324 & 0,02015 \\
\hline & $c g$ & $-4,44 \mathrm{E}+00$ & $3,78 \mathrm{E}-01$ & $-11,754$ & 0,00001 \\
\hline & $r v g$ & $-2,49 \mathrm{E}-01$ & $3,70 \mathrm{E}-01$ & $-0,674$ & 0,50016 \\
\hline & rva & $-1,93 \mathrm{E}-01$ & $3,17 \mathrm{E}-01$ & $-0,608$ & 0,54286 \\
\hline & $b$ & $1,42 \mathrm{E}-06$ & $2,69 \mathrm{E}-07$ & 5,301 & 0,00001 \\
\hline & $y$ & $-2,67 \mathrm{E}-01$ & $4,97 \mathrm{E}-01$ & $-0,538$ & 0,59086 \\
\hline & de & $1,22 \mathrm{E}+00$ & $6,67 \mathrm{E}-01$ & $1,83 \mathrm{E}+00$ & $6,66 \mathrm{E}-02$ \\
\hline & $p$ & $5,17 \mathrm{E}-01$ & $5,41 \mathrm{E}-01$ & $9,55 \mathrm{E}-01$ & $3,40 \mathrm{E}-01$ \\
\hline & $s$ & $1,52 \mathrm{E}-01$ & $4,76 \mathrm{E}-01$ & $3,20 \mathrm{E}-01$ & $7,49 \mathrm{E}-01$ \\
\hline & $n a$ & $-3,04 \mathrm{E}+00$ & $2,14 \mathrm{E}-01$ & $-14,218$ & 0,00001 \\
\hline & as & $-3,76 \mathrm{E}-01$ & $3,55 \mathrm{E}-01$ & $-1,058$ & 0,28985 \\
\hline & $a p$ & $1,10 \mathrm{E}+00$ & $5,74 \mathrm{E}-01$ & 1,919 & 0,05493 \\
\hline & ne & $-3,11 \mathrm{E}-02$ & $9,98 \mathrm{E}-02$ & $-0,311$ & 0,75548 \\
\hline & $e$ & $1,94 \mathrm{E}-02$ & $6,15 \mathrm{E}-03$ & 3,148 & 0,00165 \\
\hline
\end{tabular}




\section{CUADRO 5 (continuación)}

Estimación de la función de voto en las elecciones generales de 1996

\begin{tabular}{|c|c|c|c|c|c|}
\hline Opción electoral & Variable & Coeficiente & $\begin{array}{l}\text { Desviación } \\
\text { estándar } \\
\text { coeficiente }\end{array}$ & T-Student & $\begin{array}{c}\text { Nivel } \\
\text { significación } \\
{\left[\text { Prob }|t|>x\left({ }^{*}\right)\right]}\end{array}$ \\
\hline \multirow{16}{*}{$\mathrm{CiU}$} & $c$ & $-8,05 \mathrm{E}-01$ & 0,25614 & $-3,142$ & 0,00168 \\
\hline & $l$ & $4,49 \mathrm{E}-01$ & 0,10012 & 4,485 & 0,00001 \\
\hline & $d i$ & $1,79 \mathrm{E}-02$ & $1,24 \mathrm{E}-01$ & 0,145 & 0,88471 \\
\hline & $c g$ & $-1,58 \mathrm{E}+00$ & $4,72 \mathrm{E}-01$ & $-3,339$ & 0,00084 \\
\hline & $r v g$ & 4,09E-01 & $4,32 \mathrm{E}-01$ & 0,947 & 0,34364 \\
\hline & rva & $-5,38 \mathrm{E}+00$ & $3,48 \mathrm{E}-01$ & $-15,477$ & 0,00001 \\
\hline & $h$ & $1,83 \mathrm{E}-06$ & $3,10 \mathrm{E}-07$ & 5,885 & 0,00001 \\
\hline & $y$ & $-6,40 \mathrm{E}-02$ & $5,68 \mathrm{E}-01$ & $-1,13 \mathrm{E}-01$ & $9,10 \mathrm{E}-01$ \\
\hline & $d e$ & $1,15 \mathrm{E}+00$ & $7,50 \mathrm{E}-01$ & $1,54 \mathrm{E}+00$ & $1,24 \mathrm{E}-01$ \\
\hline & $p$ & $9,07 \mathrm{E}-01$ & $6,24 \mathrm{E}-01$ & $1,45 \mathrm{E}+00$ & $1,46 \mathrm{E}-01$ \\
\hline & $s$ & $4,15 \mathrm{E}-03$ & $5,34 \mathrm{E}-01$ & 0,008 & 0,99380 \\
\hline & na & $5,04 \mathrm{E}-01$ & $3,00 \mathrm{E}-01$ & 1,682 & 0,09254 \\
\hline & as & $-6,47 \mathrm{E}-01$ & $3,97 \mathrm{E}-01$ & $-1,631$ & 0,10294 \\
\hline & $a p$ & $-1,01 \mathrm{E}-01$ & $8,84 \mathrm{E}-01$ & $-0,114$ & 0,90947 \\
\hline & ne & $-8,11 \mathrm{E}-02$ & $1,23 \mathrm{E}-01$ & $-0,659$ & 0,50963 \\
\hline & $e$ & $1,27 \mathrm{E}-02$ & $1,02 \mathrm{E}-02$ & 1,244 & 0,21345 \\
\hline \multirow{16}{*}{$P P$} & $c$ & $2,82 \mathrm{E}-01$ & 0,22505 & 1,253 & 0,21007 \\
\hline & $l$ & $3,39 \mathrm{E}-01$ & $8,93 \mathrm{E}-02$ & 3,796 & 0,00015 \\
\hline & $d i$ & $-1,66 \mathrm{E}-02$ & $1,24 \mathrm{E}-01$ & $-0,133$ & 0,89385 \\
\hline & $c g$ & $4,87 \mathrm{E}+00$ & $4,53 \mathrm{E}-01$ & 10,757 & 0,00001 \\
\hline & rvg & $4,89 \mathrm{E}-02$ & $4,06 \mathrm{E}-01$ & 0,12 & 0,90417 \\
\hline & rva & $-8,08 \mathrm{E}+00$ & $4,10 \mathrm{E}-01$ & $-19,687$ & 0,00001 \\
\hline & $b$ & $1,62 \mathrm{E}-06$ & $3,04 \mathrm{E}-07$ & 5,32 & 0,00001 \\
\hline & $y$ & $5,83 \mathrm{E}-01$ & $5,25 \mathrm{E}-01$ & $1,11 \mathrm{E}+00$ & $2,67 \mathrm{E}-01$ \\
\hline & $d e$ & $1,08 \mathrm{E}+00$ & $7,08 \mathrm{E}-01$ & $1,53 \mathrm{E}+00$ & $1,26 \mathrm{E}-01$ \\
\hline & $p$ & $5,75 \mathrm{E}-01$ & $6,13 \mathrm{E}-01$ & $9,38 \mathrm{E}-01$ & $3,48 \mathrm{E}-01$ \\
\hline & $s$ & $-2,59 \mathrm{E}-02$ & $5,05 \mathrm{E}-01$ & $-0,051$ & 0,95908 \\
\hline & $n a$ & $-4,13 \mathrm{E}+00$ & $3,15 \mathrm{E}-01$ & $-13,12$ & 0,00001 \\
\hline & as & $-5,99 \mathrm{E}-01$ & $4,05 \mathrm{E}-01$ & $-1,48$ & 0,13893 \\
\hline & $a p$ & $8,46 \mathrm{E}-01$ & $6,46 \mathrm{E}-01$ & 1,309 & 0,19042 \\
\hline & ne & $1,24 \mathrm{E}-01$ & $1,12 \mathrm{E}-01$ & 1,105 & 0,26897 \\
\hline & $e$ & $9,16 \mathrm{E}-03$ & $1,97 \mathrm{E}-03$ & 4,656 & 0,00001 \\
\hline
\end{tabular}

$\left(^{*}\right)$ Los niveles de significación utilizados son los de la $\mathrm{N}(0,1)$.

Número observaciones $=1.883$. Grados libertad $=60$.

Logaritmo de verosimilitud $=-634,8549$. Número iteraciones $=4$.

Ji-Cuadrado (64 grados libertad) =3561,296. Nivel de significación Ji-Cuadrado = 0,00000001.

FUENTE: Elaboración propia. 
No podemos finalizar esta evaluación de las funciones de voto estimadas sin señalar que la bondad de los ajustes (ILR) es aceptable y que la capacidad predictiva, tanto a nivel global como individual (por opciones políticas), de los modelos estimados es elevada, si exceptuamos los casos de las funciones de voto de la UCD en los comicios de 1982, del PNV en las elecciones de 1996 y el CDS en las convocatorias de 1982, 1986 y 1989.

A la luz de las estimaciones realizadas, hemos elaborado un decálogo con los aspectos más reseñables que han caracterizado la decisión final de los electores españoles en la democracia.

- La consolidación del PP como alternativa de gobierno vino marcada por la influencia de factores de indole racionalista. Efectivamente, en las elecciones generales de 1993, la percepción prospectiva de la situación política fue el factor que más contribuyó a que el partido liderado por José María Aznar se erigiese en una alternativa de gobierno. Por el contrario, en los comicios de 1996 fue la capacidad de gobierno que percibían los electores españoles en el candidato popular el elemento que más favoreció en el triunfo del PP.

Nadie discute que las elecciones parlamentarias de 1993 estuvieron dominadas por la crisis económica y, sobre todo, por issues como la corrupción, que enturbiaban la percepción que los españoles tenían de la situación política. Ello explica por qué las expectativas electorales del PP aumentaban un 15,78\% entre los electores más optimistas, que presagiaban una mejoría en el ámbito político tras la celebración de los comicios.

- La capacidad de gobierno que percibian los votantes en José María Aznar fue crucial para el triunfo electoral del PP en los comicios de 1996. El tiempo que transcurrió entre 1993 y 1996 se percibió como un puente entre el ciclo gubernativo socialista y el que se atisbaba en el horizonte, presidido por José María Aznar. En ese sentido, hay quienes consideran que ese periodo estuvo dominado por la crisis del socialismo ${ }^{26}$; de ahí que la probabilidad electoral del PP aumentase un 60,38\% entre aquellos electores que percibían en el partido liderado por José María Aznar una gran capacidad para gobernar el país.

No obstante, éste no fue el único factor que posibilitó la alternancia en el gobierno tras los comicios de 1996, ya que hubo otros elementos socioestructurales que contribuyeron a la victoria del PP en estas elecciones: el hecho de que el elector fuese la persona que más ingresos aportaba al hogar, su nivel de estudios y el tamaño del hábitat ${ }^{27}$.

- La fidelidad electoral explica el realignment electoral de 1982. Efectivamente, esta variable fue la que más perjudicó las expectativas de la UCD,

${ }^{26}$ Vid. J. I. Wert (1999), «Elecciones, partidos y gobierno en la transición y la democracia», incluido en J. L. Sáez Lozano (coord.), Economía y política en la transición y la democracia.

${ }^{27}$ Véase el cuadro 5. 
mientras que en el caso del PSOE se erigió en el segundo factor que más contribuyó a su triunfo. Entre los electores más leales, es decir, entre aquellos que optaron por la misma opción política en los comicios de 1979 y 1982, la probabilidad de voto de la UCD disminuía un 5,41\%; sin embargo, la esperanza electoral del PSOE aumentaba un 23,42\%.

No obstante, este cambio en el panorama electoral español estuvo también marcado por la influencia de otros factores socioestructurales, aunque su impacto en la decisión final de los votantes fue relativamente inferior: régimen de trabajo, estado civil, género, tamaño de la familia, situación laboral y tamaño del hábitat. En las elecciones de 1982, entre los asalariados, las expectativas de voto del PSOE aumentaban un 25,95\%; en el caso de los casados, un 14,12\%; entre los varones, un 5,74\%; entre los desempleados, un 4,38\%; y entre aquellos que se declaraban católicos, un 3,38\%.

- La consolidación de la hegemonía electoral del PSOE en las elecciones de 1986 y 1989 se fundamentó en el recuerdo de voto. Uno de los rasgos que ha caracterizado al electorado socialista, y al mismo tiempo le ha permitido a esta fuerza política diferenciarse de su principal rival político en los comicios parlamentarios de 1986 y 1989, fue la fidelidad electoral de sus votantes. El recuerdo de voto de las elecciones generales ha sido el elemento que más ha influido en la decisión final de los electores socialistas: en la convocatoria de 1986, las expectativas electorales del PSOE aumentaban un 66,42\% entre aquellos votantes que habían optado en los comicios generales de 1982 por este partido; sin embargo, en las elecciones de 1989 y 1993, el impacto de esta variable descendió hasta el 62,68 y 10,25\%, respectivamente.

La lealtad de los votantes socialistas no sólo fue el principal argumento que justificó el triunfo de esta fuerza política en los comicios de 1986 y 1989, sino que también se erigió en el factor que explicó la hegemonía electoral del partido liderado por Felipe González en la segunda mitad de la década de los ochenta. A diferencia del PSOE, los votantes del PP han evidenciado una menor fidelidad: el recuerdo de voto de las elecciones generales perjudicó sus expectativas electorales, llegando incluso a ser el factor que más dañó la esperanza de voto de los populares ${ }^{28}$.

- El recuerdo de voto fue clave para el triunfo socialista en las elecciones generales de 1993. Desde 1992, las encuestas evidenciaban el descenso en intención de voto del PSOE, al mismo tiempo que aumentaban las expectativas electorales del PP. Los resultados demostraron que estos comicios fueron los más competitivos de los últimos años, al mismo tiempo que auguraban un cambio en el horizonte político de nuestro país ${ }^{29}$.

${ }^{28}$ Véanse los cuadros 2 y 3.

${ }^{29}$ Vid. J. I. Wert (1999), op. cit., incluido en J. L. Sáez Lozano (coord.), op. cit.; R. López Pintor (1998), "Las elecciones en un sistema multipartidista y plurirregional», incluido en M. Juárez (ed.), V Informe sociológico sobre la situación social en España. Sociedad para todos en el año 2000, pp. 605-628. 
Aunque los sondeos de opinión previos a las elecciones generales del 6 de junio de 1993 pronosticaban un triunfo del partido liderado por José María Aznar, sin embargo, fueron los socialistas quienes terminaron obteniendo una victoria in extremis. La influencia del recuerdo de voto fue clave, ya que favoreció las expectativas electorales del PSOE y perjudicó la esperanza de voto del PP. Efectivamente, entre aquellos electores que habían votado a estas fuerzas políticas en los comicios generales de 1989 , la esperanza de voto socialista aumentaba un $10,25 \%$, mientras que la probabilidad electoral de los populares descendía un $16,08 \%$. De todo lo anterior no debemos inferir que la fidelidad electoral fue el único elemento que propició el triunfo del PSOE en las elecciones de 1993, pues hubo otros issues como los debates televisados, cleavages como el nacionalismo, el estado de actividad, el ámbito de trabajo, el nivel de estudios y la edad, y factores como la distancia ideológica, identificación partidista y liderazgo, que también contribuyeron a la victoria socialista ${ }^{30}$.

- La función de voto del PCE/IU está dominada por la identificación partidista y la religión. Es evidente que hay dos elementos que actúan de anclaje electoral a la hora de diferenciar la función de voto del PCE/IU y las restantes fuerzas políticas que se ubican a su derecha: la importancia de la identificación partidista y el impacto del cleavage religión.

Uno de los aspectos más relevantes del comportamiento electoral de los españoles en la democracia es su escasa identificación con los partidos del espectro político (Gunther y Montero, 1994; Montero, 1994, y Montero, 1996); sin embargo, hasta ahora no se había analizado el impacto de este vínculo psicológico en la decisión final de los votantes españoles. Nuestro análisis evidencia una influencia positiva en el caso del PCE/IU: en las elecciones generales de 1986, 1989 y $1993^{31}$, a medida que los electores se identificaban más con esta fuerza política, su esperanza electoral aumentaba un 0,45 , un 4,9 y un $5,35 \%$, respectivamente. Además, ello adquiere mayor relevancia cuando se observa que ninguna de las otras opciones se han beneficiado del impacto de este factor partidista ${ }^{32}$.

A partir de las elecciones generales de 1982, el PCE/IU no sólo se ha beneficiado de la correlación positiva entre identificación partidista y voto, sino que también se ha visto afectado por la competencia electoral de naturaleza centrípeta de nuestro sistema político. Ello explica por qué las expectativas electorales de este fuerza política aumentaban a medida que los electores ubicados a su derecha se sentían más identificados con sus postulados.

Tal y como reseñamos, la función de voto del PCE/IU no sólo ha estado dominada por estos factores de identificación partidista e ideológico, sino que

${ }^{30}$ Véase el cuadro 4.

${ }^{31}$ Recordemos que en los comicios generales de 1982 y 1996 no hemos podido analizar la influencia de la identificación partidista, porque las encuestas postelectorales realizadas por el CIS no incluían ninguna pregunta que plantease esta cuestión.

32 Véanse los cuadros 2, 3 y 4. 
también ha sufrido el impacto del cleavage religioso. La secularización de la sociedad española, antes de la restauración del régimen de libertades públicas en 1976 (García Escudero, 1985), y su proyección en las preferencias ideológicas de los votantes (Montero, 1996), condicionó la decisión final de los electores. Nuestro estudio corrobora que este cleavage ha perjudicado las expectativas electorales del PCE en los comicios generales de 1982 y 1986, y de IU en 1989.

Por otra parte, el proceso de secularización y la no politización de los conflictos religiosos han favorecido el realineamiento actitudinal y creencial de los españoles (Montero, 1993); de ahí que la probabilidad de voto del PCE aumentase un $1,77 \%$ en las elecciones generales de 1986, a medida que los electores católicos eran más practicantes. En general, la izquierda se benefició de este proceso, pues las expectativas del PSOE también aumentaban un $5,31 \%$ entre los votantes más devotos. De todo lo anterior se concluye que el factor religioso ha originado una escisión, si bien es cierto que ésta se ha visto atenuada por los cambios que se han producido en la sociedad española.

- El cleavage nacionalista es el factor más determinante a la hora de adoptar su decisión los votantes de CiU y PNV. Efectivamente, si analizamos las funciones de voto de estas dos fuerzas políticas de Cataluña y País Vasco observamos que, entre los electores nacionalistas, su esperanza electoral aumenta en todas las convocatorias, si exceptuamos el caso del PNV en los comicios generales de 1986, en donde el impacto de este cleavage fue superado por otros factores de índole partidista, racional... ${ }^{33}$. La pérdida de protagonismo de la variable nacionalista vino marcada por la excepcionalidad que vivió el sistema político del País Vasco, tras la división que se gestó en el PNV: en 1984, Carlos Garaicoetxea dimitió como Lendakari y fundó Eusko Alkartasuna (EA) (Llera, 1998).

En el caso de $\mathrm{CiU}$, al no producirse esta escisión, la influencia de este cleavage ha evidenciado un comportamiento regular a lo largo de la democracia. Incluso en los comicios de 1986, entre los votantes catalanes, las expectativas electorales del partido liderado por Jordi Pujol alcanzaron la cota del 55,55\%.

- La función de voto del CDS estuvo dominada por factores coyunturales: la influencia de las encuestas en los comicios de 1986 y el recuerdo de voto en las elecciones de 1989. El partido centrista, liderado por Adolfo Suárez, irrumpió en los comicios de 1982; pero fue en las elecciones generales de 1986 cuando alcanzó su máxima representación parlamentaria. Los factores que más influyeron en la decisión final de los votantes del CDS fueron las encuestas, el voto prospectivo y la religión.

Efectivamente, las encuestas publicadas en los días previos a los comicios celebrados el 22 de junio de 1986 presagiaban un ascenso electoral del partido

33 Véase el cuadro 2. 
centrista. Al final, este issue se erigió en un factor determinante del éxito obtenido por el CDS en estas elecciones, ya que entre aquellos votantes que reconocían tener noticias de los pronósticos electorales, la probabilidad de voto de esta fuerza política aumentaba un 19,79\%. En ese sentido, hay que interpretar este hecho como un efecto demostración, en el que los buenos resultados que anticipaban los sondeos de opinión alimentaban las expectativas de voto del partido liderado por Adolfo Suárez.

Tal y como presagiaban las elecciones al Parlamento Europeo celebradas el 15 de julio de 1989, el CDS entró en una fase de declive irreversible. Quizás la derrota electoral en los comicios generales celebrados el 29 de octubre de este año habría sido mayor si el recuerdo de voto, la religión, el nivel de estudios y el tamaño del hábitat no hubiesen influido positivamente en la decisión final de los electores centristas ${ }^{34}$.

- Las elecciones generales de 1982 culminaron la pos-transición y el cambio: la oposición pasó a gobernar, pero el gobierno no se convirtió en oposición ${ }^{35}$. Quizás ésta sea una forma plástica de expresar la debacle electoral de la UCD en los comicios de 1982, y la transformación que registró el espectro político español. Una de las razones que incitaron a más de un $80 \%$ del electorado de la UCD a no renovarle su confianza en los comicios de 1982 fue el recuerdo de voto de las generales de 1979. Entre aquellos electores que habían votado a la coalición en estos comicios, la esperanza electoral de la coalición disminuía un 5,41\%. Tras este factor se situaban una serie de variables socioestructurales que también perjudicaron las expectativas de la UCD: en el caso de los pensionistas y entre los hombres, la probabilidad de voto del partido centrista disminuía un 4,24\%, entre los desempleados caía un 4,15\%, en el colectivo de los casados disminuía un 4,10\%, entre los votantes de Cataluña y País Vasco descendía un 3,65\%, y en el caso de los asalariados caía un 2,44\%.

La UCD tan sólo encontró un cierto consuelo electoral en el cleavage religión y algunos factores socioestructurales como el nivel de estudios y la edad. Entre aquellos votantes que se declaraban católicos en 1982, las expectativas de la coalición centrista aumentaban un 1,11\%. Por otra parte, a medida que los votantes poseían un mayor nivel de educación o tenían más edad, la probabilidad de voto de la UCD crecía un 0,39 y $0,18 \%$, respectivamente.

- Del «realignment» de las elecciones generales de 1982 al cambio de los comicios de 1996. Las convocatorias electorales de 1982 y 1996 depararon un cambio de gobierno: en 1982 el PSOE obtuvo mayoría absoluta, mientras que en 1996 el PP logró una mayoría relativa. Los análisis realizados hasta ahora concluyen que hubo un realignment en la convocatoria de 1982, pero en ningún caso estas elecciones pueden calificarse de críticas (Key, 1955), ya que no

${ }^{34}$ Véase el cuadro 3.

${ }^{35}$ R. López Pintor y J. I. Wert (1984), La vida política. 
se produjo un realignment ideológico ${ }^{36}$. Por otra parte, hay quienes mantienen que la victoria del PP en las elecciones de 1996 fue efímera, ya que los populares no conquistaron el voto de aquellos electores que tradicionalmente venían optando por el PSOE o los partidos nacionalistas (PNV y CiU); más bien, los resultados de estos comicios evidenciaron que estábamos ante un cambio coyuntural, ya que el éxito electoral del partido liderado por José María Aznar se fundamentó en issues como la corrupción, la judicialización de la vida política.... ${ }^{37}$.

En ese sentido, las funciones de voto estimadas corroboran el realignment de 1982 y el cambio político de 1996. El triunfo del PSOE en los comicios de 1982 se apoyó en factores estructurales, con una base social muy fuerte: el régimen de trabajo, el estado civil, el género, el tamaño de la familia, la situación laboral, el tamaño del hábitat y la religión. Tan sólo hubo un elemento coyuntural que favoreció la victoria del PSOE en 1982: el recuerdo de voto de las generales de $1979^{38}$.

Si comparamos las funciones de voto del PP y PSOE en las elecciones generales de 1996, concluimos que los populares basaron su victoria en tres factores estructurales (persona que más ingresa, nivel de estudios y tamaño del hábitat) y en la capacidad de gobierno que percibían los electores en José María Aznar (coyuntural). La influencia de este issue fue el principal aliado del PP y el gran enemigo electoral del PSOE en los comicios de 1996. En cualquier caso, no debemos olvidar que la función de voto socialista seguía teniendo una base muy sólida, ya que el estado de actividad, el ámbito de trabajo, el componente nacionalista, la edad, el género, la situación laboral y el régimen de trabajo favorecieron las expectativas de voto del partido liderado por Felipe González en los comicios de 1996. Al margen de estos factores, la distancia ideológica fue otro elemento estructural que benefició más a los socialistas; sin olvidar tampoco el fuerte impacto de variables coyunturales como el recuerdo de voto (autonómicas y generales), la campaña electoral y el liderazgo, que favorecieron más al PSOE.

\section{CONCLUSIONES}

De la lectura de los epígrafes anteriores se infiere que la función de voto es un enfoque comprehensivo del comportamiento electoral, ya que nos permite analizar conjuntamente cómo influyen los factores ideológicos, las variables

${ }^{36}$ Vid. R. Gunther, G. Sani y G. Shabad (1986), El sistema de partidos politicos en España. Génesis y evolución, pp. 448-450; G. Sani (1986), Los desplazamientos del electorado: anatomía del cambio, pp. 1-26; R. Gunther (1986), El realineamiento electoral del sistema de partidos de 1982, pp. 27-70; L. Paramio (1998), Clase y voto: estructuras y preferencias.

37 Vid. J. Rodríguez Menés (1998), The Spanish labyrinth: class and nationalism in contemporary Spain. Medium-run polarization in young democracies.

${ }^{38}$ Véase el cuadro 1. 
racionalistas y las cuestiones socioestructurales en la decisión final del elector. Ello nos ha obligado a asumir que el objetivo de cualquier votante es maximizar su nivel de utilidad electoral.

Del análisis empírico sobre la democracia española se concluye que un factor de índole racionalista, como es la capacidad de gobierno que percibían los votantes en José María Aznar, fue crucial para el triunfo electoral de los populares en los comicios de 1996. Por otra parte, no debemos olvidar que la consolidación del PP como alternativa de gobierno en las elecciones de 1993 también estuvo marcada por la influencia de variables de carácter racional: percepción prospectiva de la situación política y económica. Si a todo ello le agregamos que la renta y el desempleo (variables socioeconómicas) favorecieron las expectativas electorales de los populares en estas dos convocatorias electorales, concluiremos que el voto económico ha sido clave en la función de voto del PP.

En la orilla izquierda, el PSOE no sólo era penalizado por el voto económico, sino que también sufría el castigo de los votantes racionalistas: éstos no percibían que pudiese seguir gobernando, y tampoco creían que fuese capaz de mejorar la situación económica y política tras los comicios de 1996.

El realignment de las elecciones generales de 1982 facilitó la llegada del PSOE al gobierno de la nación. Su triunfo se debió a factores estructurales, y más concretamente de carácter social (régimen de trabajo, estado civil, género, tamaño de la familia, situación laboral, tamaño del hábitat y religión). Tan sólo hubo un elemento coyuntural que contribuyó a la victoria del PSOE en 1982, y que se ha erigido en uno de sus principales aliados de la función de voto socialista a partir de esta convocatoria electoral: el recuerdo de voto de las elecciones generales.

Es evidente que el realignment de 1982 no hubiese sido posible sin la debacle electoral de la UCD, coalición que había gobernado desde las primeras elecciones democráticas celebradas en 1977. Si tuviésemos que destacar las razones que incitaron a más de un $80 \%$ del electorado de la UCD a no renovarle su confianza en los comicios de 1982, destacaríamos la infidelidad de los mismos, además de otras variables socioestructurales que también perjudicaron las expectativas electorales de la coalición: estado de actividad, género, situación laboral, estado civil, nacionalismo y régimen de trabajo.

La hegemonía electoral del PSOE en las elecciones de 1986 y 1989 se fundamentó en el recuerdo de voto. La lealtad de sus electores no sólo fue una cuestión clave, sino que también se erigió en el principal aliado estratégico, que evitó la derrota del partido liderado por Felipe González en los comicios generales de 1993 .

La función de voto del PCE/IU ha estado dominada por la influencia positiva de la identificación partidista y el impacto negativo del cleavage religión. Por el contrario, en los casos de CiU y PNV, el cleavage nacionalista ha sido el factor más determinante, si exceptuamos el caso del partido vasco en los comicios generales de 1986 .

No podemos finalizar sin señalar que la irrupción del CDS en los comicios 
de 1982, su posterior consolidación en las elecciones generales de 1986 y su ulterior declive en la convocatoria de 1989 han estado marcados por factores estrictamente coyunturales: la influencia de las encuestas en 1986 y el recuerdo de voto en 1989.

\section{BIBLIOGRAFÍA}

ACHen, Ch. H. (1975): «Mass political attitudes and the survey response», American Political Science Review, vol. 69.

Álvarez, R. M., y Nagler, J. (1995): «Economics, issues and the Perot candidacy: voter choice in the 1992 presidential election", American Journal of Political Science, vol. 39, n.o 3.

Ashenfelter, O., y Kelley, S., Jr. (1975): «Determinants of participation in presidential elections", Journal of Law and Economics, vol. 18.

Barzel, Y., y Silbergerg, E. (1973): «Is the act of voting rational», Public Choice, vol. 16.

BLACK, D. (1958): Theory of committees and elections, New York: Cambridge University Press.

Campbell, A.; Converse, P. E.; Warren, E., y Stokes, D. E. (1960): The American voter, New York: Wiley and Sons.

DeclercQ, E.; Hurley, T. L., y Luttberg, N. R. (1975): «Voting in American Presidential elections: 1956-1972», American Politics Quarterly, vol. 3.

Downs, A. (1957): An economic theory of democracy, Harper and Row.

Durden, D., y GAYNOR, P. (1987): "The rational behavior theory of voting participation: Evidence for the 1970 and 1982 elections", Public Choice, vol. 53.

Fiorina, M. F. (1977): «An outline for a Model of Party Choice», American Journal of Political Science, vol. XXI, n. 3 .

García Escudero, J. M. ${ }^{a}$ (1985): "La sensibilidad del catolicismo español desde los años cincuenta hasta el momento actual», Cuenta y Razón, n. ${ }^{\circ} 20$.

GoldberG, A. S. (1966): «Dicerning a causal pattern among data on voting behavior», American Political Science Review, vol. 60.

Gracia DíEZ, M. (1988): "Modelos con variable dependiente cualitativa y de variación limitada», Cuadernos Económicos ICE, vol. 39.

Greene, W. H. (1998): Análisis econométrico, Prentice Hall.

GunTHER, R. (1986): "El realineamiento electoral del sistema de partidos de 1982», incluido en J. J. Linz y J. R. Montero (eds.), Crisis y cambio: electores y partidos en la España de los años ochenta, Centro de Estudios Constitucionales.

Gunther, R., y Montero, J. R. (1994): «Los anclajes del partidismo: Un análisis comparado del comportamiento electoral en cuatro democracias del sur de Europa», incluido en P. del Castillo (ed.), Comportamiento político y electoral, Centro de Investigaciones Sociológicas.

Gunther, R.; SANI, G., y SHABAD, G. (1986): El sistema de partidos políticos en España. Génesis y evolución, Centro de Investigaciones Sociológicas, Siglo XXI.

Harrop, M., y Miller, W. L. (1990): Elections and voters. A Comparative Introduction, Macmillan Education.

IYENGAR, S. (1990): «Shortcus to political knowledge: The role of selective», incluido en J. A. Farejonh y J. H. Kuklinski (eds.), Information and democratic processes, University of Illinois Press.

JACKSON, J. E. (1975): «Issues, party choice and presidential votes», American Journal of Political Science, vol. 19.

KaIERO, A. (ed.): Valores y estilos de vida, Universidad de Deusto.

KeY, V. O., Jr. (1955): «A theory of critical elections», The Journal of Politics, vol. 17.

Kramer, G. H. (1971): "Short-term fluctuations in Us voting behavior», American Political Science Review, vol. 65. 
Kramer, G. H. (1983): «The ecological fallacy revisited: Aggregate-versus individual-level findings on economics and elections, and sociotropic voting», American Political Science Review, vol. 77.

LEWIS-BECK, M. S. (1990): Economics and elections: The major western democracies, Ann Arbor: The University of Michigan Press.

LinZ, J. J., y Montero, J. R. (eds.): Crisis y cambio: electores y partidos en la España de los años ochenta, Centro de Estudios Constitucionales.

LIPSET, S. M., y RoKKAN, S. (1967): Party systems and voter alignments, New York: Macmillan.

LÓpez Pintor, R., y Wert, J. I. (1984): «La vidad política», incluido en R. López Pintor y R. Hagemeyer (eds.), España: Balance 1983, Oycos-Fundación Konrad Adenauer.

LLERA RAMO, F. J. (1998): «Pluralismo y gobernabilidad en Euskadi (1980-1994)», incluido en M. Alcántara y A. Martínez (eds.), Las elecciones autonómicas en España, 1980-1997, Centro de Investigaciones Sociológicas.

Markus, G. B., y Converse, P. E. (1979): «A dinamic simultaneous equation model of electoral choice", American Political Science Review, vol. 73.

Matsusaka, J. G., y Palda, F. (1993): "The Downsian voter meets the ecological fallacy», Public Choice, vol. 77.

Montero, J. R. (1993): «Las dimensiones de la secularización: religiosidad y preferencias políticas en España", incluido en R. Díaz-Salazar y S. Giner (eds.), Religión y sociedad en España, Centro de Investigaciones Sociológicas.

- (1994): «Sobre las preferencias electorales en España: fragmentación y polarización (19771993)", incluido en P. del Castillo (ed.), Comportamiento político y electoral, Centro de Investigaciones Sociológicas.

- (1996): «Elecciones y comportamiento electoral: dimensiones, factores y reglas», incluido en J. Tussel, E. Lamo de Espinosa y R. Pardo (eds.), Entre dos siglos. Reflexiones sobre la democracia española, Alianza Editorial.

Nie, N. H.; Verba, S., y PetrociK, J. R. (1976): The changing American voter, Harvard University Press.

Page, B. I., y Brody, R. A. (1972): «Policy voting and the electoral process: The Vietnam war issues», American Political Science Review, vol. 66.

PAge, B. I., y Jones, C. C. (1979): «Reciprocal effects of policy preferences, party loyalties and the vote», The American Political Science Review, vol. 73.

PARAMIO, L. (1998): Clase y voto: estructuras y preferencias, Ponencia presentada en el VI Congreso Español de Sociología, A Coruña.

Rabinowitz, G., y MacDonald, S. E. (1989): «A directional theory of issue voting», American Political Science Review, vol. 83.

Rahn, M. R.; Aldrich, J. H.; Borgida, E., y Sullivan, J. L. (1990): "A social-cognitive model of candidate appraisal", incluido en J. A. Farejonh y J. H. Kuklinski (eds.), Information and democratic processes, University of Illinois Press.

Riker, W. H., y Ordeshook, P. (1968): "A theory of the calculus of voting», American Political Science Review, vol. 62.

Rodríguez Menés, J. (1998): The Spanish labyrinth: class and nationalism in contemporary Spain. Medium-run polarization in young democracies, Ponencia presentada en el VI Congreso Español de Sociología, A Coruña.

Ruiz Olabuenaga, J. I. (1994): "Los estilos de vida como empatías de participación política», incluido en A. Kaiero (ed.), Valores y estilos de vida, Universidad de Deusto.

SÁEZ LozAno, J. L. (2000a): Economía y política en la Transición y la Democracia, Fundación FAES.

- (2000b): «Estabilidad política y economía en la democracia española (1976-2000)», Estudios Politicos, n. ${ }^{\circ} 110$.

- (2000c): «Economía y democracia en España», Claves de la Razón Práctica, n. ${ }^{\circ} 107$.

- (2001a): "Economic voting in Democracy, The Spanish case», Économie Appliquée, tome LIV.

- (2001b): El voto económico en España. Hacienda Pública Española, vol. 157 (2). 
SÁEZ LozAnO, J. L. (2001c): «Las elecciones del 12-M en perspectiva: Una interpretación estratégica», Revista de Estudios Políticos, n. ${ }^{\circ} 114$.

- (2002a): «Los ciclos políticos de la economía española (1975-2000). Información Comercial Española», Revista de Economía, n. 796.

- (2002b): «La función del voto económico: El caso de España», El trimestre Económico (próximo).

SANI, G. (1986): «Los desplazamientos del electorado: anatomía del cambio», incluido en J. J. Linz y J. R. Montero (eds.), Crisis y cambio: electores y partidos en la España de los años ochen$t a$, Centro de Estudios Constitucionales.

Schuman, M. A., y Pomper, G. M. (1975): «Variability in electoral behavior: Longitudinal perspectives from causal modeling», American Journal of Political Science, vol. 19.

SHAPIRO, M. J. (1969): «Rational political man: A synthesis of economic and social-psychological perspectives», The American Political Science Review, vol. 63.

Siegrried, A. (1913): Tableau politique de la France de L'Ouest sous la Troisieme Republique, Colin.

Sniderman, P. M.; Glaser, J. M., y Griffin, R. (1990): «Information and electoral choice», incluido en J. A. Farejonh y J. H. Kuklinski (eds.), Information and democratic processes, University of Illinois Press.

VAllés, J. M. (1990): "Proceso electoral, comportamiento electoral y sistema político», Revista del Centro de Estudios Constitucionales, vol. 5.

WeRT, J. I. (2000): «Elecciones, partidos y gobierno en la Transición y la Democracia», incluido en J. L. Sáez Lozano, Economía y politica en la Transición y la Democracia, Fundación FAES.

Whitten, G. D., y Palmer, H. D. (1996): «Heightening comparativists' concern for model choice: voting behavior in Great Britain and the Netherlands", American Journal of Political Science, vol. 40 , n. ${ }^{\circ} 1$.

\begin{abstract}
In this study we raise the need to analyse, from an all-embracing perspective, the decision of voters. Up until now, the theory of electoral conduct offered partial explanations of how voters position their final decision: party identification, rationalist view and the approaches raised by the social structure.

Our aim is to specify a function of voting that contains these three approaches. In order to do this, we must assume that voters can choose between a set of political options, and that the decision observed reveals which is the alternative that proves to be most useful. Since there are more than two parties, we are obliged to propose a multiple, discrete choice model. In accordance with available information, we have specified a multinomial logit model.

Starting out from estimated voting functions, we have drawn up a decalogue of the vote in Spanish democracy, with a view to synthesizing the principal features that have characterized the electoral conduct of Spaniards throughout this period.
\end{abstract}

